\title{
Constitutive equation for concrete creep and shrinkage based on thermodynamics of multiphase systems
}

\author{
Z. P. BǍ̌ANT (1)
}

\section{RÉSUMÉ}

L'action des variations d'humidité et de température sur le béton, compte tenu des facteurs de dimension et de forme, ainsi que de la répartition des contraintes est traduite par l'équation fondamentale du fluage, du retrait et de la dilatation thermique. La pâte de ciment et le béton sont étudiés en tant que matériaux composites multiphases dans lesquels les conditions d'équilibre, tant statique que thermodynamique, doivent être considérées.

\section{SUMMARY}

The constitutive equation for creep, shrinkage and thermal expansion, which reflects correctly the effect of variable humidity and temperature, including the effect of size, shape and stress distribution, is derived. Cement paste and concrete are treated as a multi-phase composite material, in which both the static and thermodynamic conditions of equilibrium must be considered.

\section{NOTATIONS}

$a_{\mathrm{T}}, a=$ rate constants for the local microscopic diffusion in eq. (16), (40) and (45);

$b_{\mathrm{T}}, b=$ rate constants for the macroscopic diffusion in eq. (7)-(8), (45);

$c, \mathrm{C}=$ diffusion constants for adsorbed layers, defined by $(7 a),(7 b)$;

$\bar{d}=$ average effective distance for microscopic diffusion (volumetric) (eq. 16), $\bar{d}^{\prime}=$ similar value for deviatoric diffusion;

$e_{i j}=$ total strain deviator;

$e_{d_{i j}}=$ deviator of the change of thickness $\bar{\delta}_{d}$ of hindered layers;

$$
\text { 22); }
$$

$f_{a}, f_{d}=$ area factor for $p_{a}$ or $p_{d}$, respectively (eq. 12

$h=$ humidity $=$ relative vapor pressure (in the pores inside);

$h_{e q}=$ equivalent humidity defined after eq. (49);

$h_{e x}=$ humidity of the external at mosphere (ambient);

$h_{s}=$ humidity at self-desiccation of a sealed sample (eq. 5);

(1) Ph. D., Doc., Ing., Associate Professor, The Technological Institute, Northwestern University, Evanston, Illinois, U.S.A. $h_{o}=$ time decrease of humidity at $\mathrm{T}_{o}$ for which the volume change without stress is zero (eq. 20);

$k, k^{\prime}=$ slopes of the desorption and sorption isotherms (eqs. $3,5,41$ );

$n=$ exponent in eq. (10);

$p=$ pressure, less the atmospheric pressure 1 atm;

$p_{a}, p_{\nu}, p_{s}=p$ in free adsorbed layers (eq. 1), in vapor, or in capillary water (eq. 2a);

$p_{d}=p$ in the hindered adsorbed layer, $p_{d}=$ its average value (eqs. 2,12 );

$q=$ activation energy for hydration (apparent), eq. (52);

$r_{1}, \quad r_{2}=$ principal curvature radii of capillary menisci;

$s_{i j}=$ total stress deviator;

$s_{d_{i j}}=$ deviator of the stress in hindered layers;

$t=$ time, or age of concrete;

$t_{e}=$ equivalent curing period, defined after eq. (4);

$u=$ displacement in the sense of $x$;

$v=$ specific volume $=(\text { mass density })^{-1}$;

$v_{a}, v_{d}, v_{c}, v_{v}=v$ for free, or hindered adsorbed layer, capillary water and vapor, respectively; $v_{c}=1 \mathrm{~cm}^{3} / \mathrm{g}$;

$w=$ total mass of water (usually per unit volume of porous material or per unit surface); 
$w_{e}, w_{n}=$ evaporable and non-evaporable water at a given $\mathrm{T}$;

$w_{a}, w_{d}, w_{c}=w$ corresponding to $v_{a}, v_{d}, v_{c}$

$x, y, z$ or $x_{1}, x_{2}, x_{3}=$ cartesian coordinates;

$\mathrm{B}=$ coefficient of water transmission at the surface, eq. (9);

$G=$ Gibbs' free energy (eq. A1) or shear modulus (eq. 40);

$G^{1}=$ partial value of $G$ per unit of mass;

$\mathrm{G}_{a}, \mathrm{G}_{b}, \mathrm{G}_{c}, \mathrm{G}_{d}, \mathrm{G}_{f}, \mathbf{K}_{a}, \mathbf{K}_{b}, \mathbf{K}_{c}, \mathbf{K}_{d}, \mathbf{K}_{f}, \mathbf{K}_{h}=$ shear moduli and volume moduli for models in figures $6 a$, $5 a$ (defined by eqs. 23, 24, 27, 40, 18);

$\mathbf{K}=$ elastic volume modulus, eq. (27);

$\mathrm{L}=$ volume stress memory function, cq. (32), (32a), $(32 e)$;

$\mathrm{L}_{a}=$ humidity memory function, eq. $(32 b),(32 c)$, $(32 f)$;

$\mathrm{L}_{\mathrm{T}}=$ temperature memory function, eq. (51), (51a);

$\mathrm{M}=$ molecular weight of water;

$P_{d}=$ disjoining pressure $=p_{d}-$ pressure in the free layer of equal thickness (eq. (A5);

$\mathrm{Q}, \mathrm{Q}^{\prime}=$ activation energies (enthalpies) in eq. (45);

$\mathbf{R}=$ universal gas constant;

$\mathrm{S}=$ entropy;

$\mathrm{T}, \mathrm{T}_{o}=$ absolute temperature and chosen reference temperature;

$\mathrm{V}=$ volume;

$\alpha_{b}, \alpha_{c}, \alpha_{d}, \alpha_{o}, \alpha_{1}=$ thermal expansion coefficients, eq. (47)-(49);

$\beta_{\mathrm{T}}=$ relative hydration rate defined by eq. (4);

$\beta=\beta_{\mathrm{T}}$ at reference temperature, eq. (52);

$\beta_{a}=$ volume compressibility of adsorbed water, eq. (14); $\gamma=$ surface tension, eq. (2a) (or shear strain);

$\delta_{a}, \delta_{d}=$ average thickness or free, or hindered adsorbed layer $\left(\delta_{a}=w_{a} / v_{a}, \delta_{d}=w_{d} / v_{d}\right)$;

$\bar{\delta}_{d}=$ average total thickness of all layers intersecting a unit length (eq. 15);

$\varepsilon=$ total volume strain $=\left(\varepsilon_{11}+\varepsilon_{22}+\varepsilon_{33}\right) / 3$;

$\varepsilon_{i j}=$ total strain tensor;

$\varepsilon_{d}=$ volume component of the change of thicknes $\mathbf{s}$ $\bar{\delta}_{d}$ of hindered layers, $d \varepsilon_{d}=d \bar{\delta}_{d}$;

$\varepsilon_{o}^{s}, \varepsilon^{c}=$ free shrinkage (eq. $32 b$ ) and creeps train defined before eq. $(22 a)$;

$x=$ hygrothermic coefficient, defined by eq. (41);

$\mu=$ chemical potential (eq. A7);

$\sigma, \sigma_{a}=$ total volume stress, and volume stress in the fluid (defined in $§ 5.2$ );

$\sigma_{d}, \sigma_{a d}=$ actual volume stress in hindered layers and its theoretical value needed for thermodynamic equilibrium at a given $p_{a}$ (eqs. 12, 17, 20);

$\varphi, \psi($ or $\tilde{\varphi})=$ rate of creep constants for volume and deviatoric deformation, eq. $(28),(25),(40),(38)$ (or eq. 29);

$\varphi_{d}, \psi_{d}=$ rate constants for microscopic volume and deviatoric diffusion, eq. (17), (19), (37);

$\tau, t^{\prime}=$ time as integration variable, or time at load application (also $\tau=$ shear stress);

$\tau_{i}=$ retardation time for the $i$-th unit in figure $5 b$ $=\tilde{\varphi}_{i}^{-1}$;

$\Delta$ denotes increments during time step $\Delta t$;

Subscript $i$ in $f_{d i}, \varphi_{i}, \mathbf{K}_{c i}, \mathbf{K}_{d i}, \mathbf{K}_{f i}, \sigma_{a d i}$ denotes values of $f_{d}$ etc. for the $i$-th unit in the chain in figure $5 b$;

$k p=$ kilopond $=$ force kilogram, $\AA=$ angström $=10^{-7}, \mathrm{~mm}, \quad \boldsymbol{u}=(u)^{\cdot}=\partial u / \partial t, \sim$ proportionality sign, $\approx$ " approximately equal", $\rightarrow$ c tending to", $\leftarrow$ "6sign"

\section{INTRODUCTION}

At the present time, the stress-strain law of creep and shrinkage of concrete is known sufficiently well only for invariable humidity and temperature conditions during the process, although some estimates have been established for predicting the effect of various levels of temperature and humidity, and the closely related effect of size, shape and stress distribution [1-20]. Under these invariable conditions a purely phenomenological theory can be developed even without a proper understanding of the physical nature of the phenomenon, by simply using the principle of superposition in time, or the viscoelastic rheological models of aging bodies [21-7, 27, 8]. However, certain types of modern structures, especially the concrete structures for nuclear reactors, require a much deeper knowledge of creep, shrinkage and thermal expansion, including the conditions of variable humidity and temperature. In this case it seems necessary to base our model on the knowledge of the internal microstructure of cement paste and understanding of the creep mechanism.

The effect of humidity upon creep and shrinkage, along with the fact that completely dried specimens do not exhibit any significant creep [28-31] at low stress levels, suggests that the main source of this phenomenon is the evaporable water. This hypothesis is justified by the present knowledge about the structure of cement paste [32-34]. The role of evaporable water was already recognized by Lynam [35] and Freyssinet [92]. Later many investigators rejected this idea, since a satisfactory explanation had not been found for various aspects, such as creep in torsion and bending, separability of creep and shrinkage, the difference between the losses of water during shrinkage and creep of equal magnitude, irreversible creep and shrinkage, the increase of creep of predried samples with humidity [29-31,12] contradicting the idea of drying creep [16], etc. The load-bearing ability of hindered adsorbed water layers and their role in creep and shrinkage was first mathematically analyzed by Hrennikof [37], although verbal remarks on it may be found in earlier literature [34, p. 589 with references to Carlson and Lynam, or 95]. In terms of thermodynamics this effect was first investigated and formulated by Powers $[32,36,38]$ who based his theory on an especially thorough knowledge of cement paste structure. 
In this study we shall start from the basic ideas of Powers [32, 36, 38] (and Hrennikof [37]), endeavouring to establish a complete model for the macroscopic constitutive equation. We shall treat the cement paste and concrete as a multi-phase medium, the theory of which has been developed until now only in soil mechanics [39]. Besides the conditions of static equilibrium between phases we shall have to introduce the conditions for thermodynamic equilibrium of various phases of water and investigate the microscopic local diffusion of water. Although the delayed deformation response will be described only in terms of adsorbed water, our constitutive equation will also admit the effect of chemically bound evaporable water and capillary water, the effect of dissolution of matter under load and recrystallization, as well as viscosity (sliding) in the cement gel (see Appendix C8-10). In addition to this, we must study briefly the macroscopic diffusion in concrete, and the humidity distribution. At the end we shall present the results of computer analyses of various tests of creep, shrinkage and thermal expansion according to our constitutive equation (*).

Throughout all this study we shall consider simultaneously cement paste and concrete. Their behavior is qualitatively different but quantitatively same (see Appendix C1).

Before starting the analysis let us introduce some basic facts about the structure of Portland cement paste [32-34]. It may be described as a multi-phase porous material whose solid part consists of hydrated cement and unhydrated cement grains, made up mostly of crystalline components. The densest possible form (porosity 0.28 ) of completely hydrated cement paste is called cement gel which is predominantly amorphous but consists mainly of quasi-crystalline (and some crystalline) strongly hydrophylic particles of colloidal dimensions and laminar form (probably in shape of rolled tubes [40]). The porosity of the paste is usually between 0.40 and 0.55 . The average thickness of laminae is about. $30 \AA$, the average width of pores is about $15 \AA$ (the minimum may be $2 \AA$ ). The internal surface area is about $500 \mathrm{~m}^{2}$ per $\mathrm{cm}^{3}$ of material.

\section{EqUilibrium of EVAPORABLe WATER in CEMENT PASTE}

\subsection{Adsorbed water and its conditions of thermodynamic equilibrium}

Near a solid surface the van der Waals forces affect the movement of the adjacent molecules of water vapor and retain them at the solid surface for a certain "lingering time" (ranging from 10-12 sec to perhaps $2 \sec [36,41])$. These molecules form thin adsorbed water layers. The specific mass $w_{a}$ of water adsorbed per unit solid surface, and thus the average thickness $\delta_{a}$ of the adsorbed layer, increase with humidity $[32,42,43]$ and for $h \rightarrow 1$ it is reasonable to assume a maximum thickness of about 5 molecules, i.e. about $13 \AA$. At $25{ }^{\circ} \mathrm{C}$ a monomolecular layer, $\delta_{a}=2.63 \AA$ [32], is achieved for $h=0.12$ (two molecule thickness at $h=0.51$, half molecule average thickness at $h=0.03$ ). Thermodynamic equilibrium requires that [36] (under certain simplifying assumptions, see Appendix A).

$$
p_{a}=\frac{R T}{M_{v_{a}}} \ln h \quad \text { where } h=\frac{p_{V}}{p_{\text {sat }}} \leqslant 1 \quad \text { (1) }
$$

where $p_{v}=$ vapor pressure, $p_{\text {sat }}=$ saturated vapor pressure depending on temperature $\mathrm{T}, p_{a}=$ pressure in the adsorbed water, less the atmospheric pressure, $1 \mathrm{~atm}\left(p_{a}\right.$ is always negative, i.e. tension), $\mathrm{R}=$ universal gas constant, $M=$ molecular weight of water, $v_{a}=$ specific volume of adsorbed water which is approximately equal to that of capillary water, $v_{c}\left(\delta_{a}=w_{a} v_{a}\right)$.

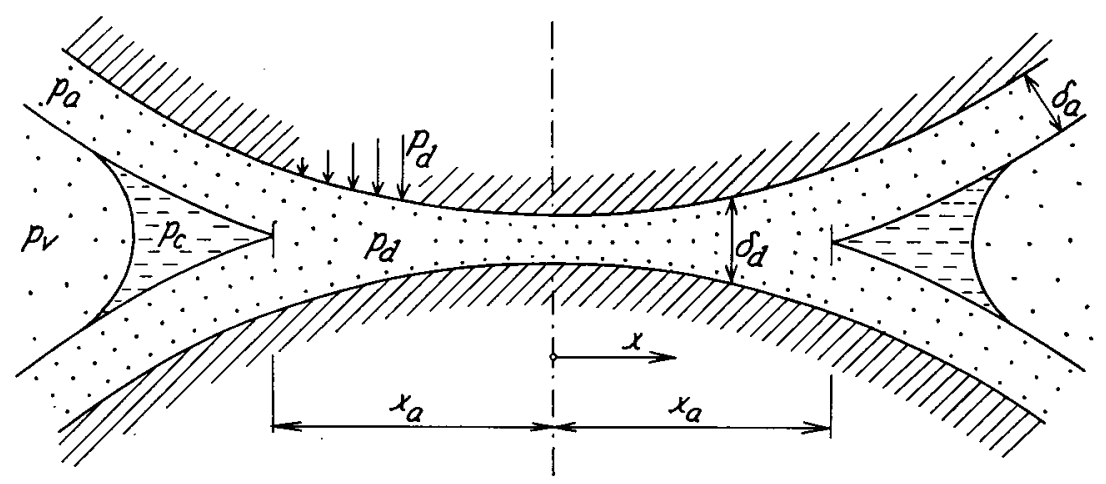

FIG. 1. - Idealized hindered
adsorbed water layer. (The
change of its thickness
along the layer is exagge-
rated. Actually it has to
be imagined much longer.)

Eq. (1) is valid only as long as $v_{a}$ may be approximately considered invariable with $h$. It is invalid for $h<0.12$. At $25^{\circ} \mathrm{C}, \mathrm{RT} / \mathrm{M} v_{a}=1360$ atm (a value which equals $p_{a}$ at $h=0.366$ ).

In a narrow gap (Fig. 1) which is thinner than about $26 \AA$, above a certain humidity the full thickness of the two adsorbed layers at the opposite solid surfaces cannot be accomodated. In this

(*) Further development of the present theory is given in the author's report no SESM 69-11, ". Thermodynamic theory of concrete deformation at variable temperature and humidity ", Department of Civil Engineering, University of California, Berkeley, August 1969 (added in proof). 
case we speak of hindered adsorption, in distinction to the previous case of free or unhindered adsorption [36]. In state of thermodynamic equilibrium the following must hold (see Appendix A)

$$
p_{d}=p_{a}
$$

where $p_{d}=$ pressure in the hindered layer (less $1 \mathrm{~atm}$ ). Since the pressure in the adsorbed layer is a function of its thickness which is different in the hindered and adjacent free layers, eq. (2) requires that an additional pressure $\mathrm{P}_{d}$, called disjoining pressure, be applied on the solid surfaces in order to make the total pressure $p_{d}$ equal to $p_{a}$ and keep the gap thickness constant. Thus eq. (2) expresses the load-bearing ability of the hindered adsorbed water [37,36], a fact which is crucial in Hrennikof's and Powers' theory of creep. The gap to be entered by hindered layers must have at least one molecular thickness $(2.63 \AA)$. It becomes full of adsorbed water when the adjacent free layers in equilibrium are about a half molecule thick, i.e. $h \approx 0.03$ (at $\mathrm{T}=25^{\circ} \mathrm{C}$ ). Below this humidity we cannot speak of hindered adsorption.

The condition of thermodynamic equilibrium of capillary water (Kelvin) and the differential condition of equilibrium at the interface with vapor (Young and Laplace) provide

$$
p_{c}=p_{a}, \quad p_{c}=-\gamma\left(r_{1}^{-1}+r_{2}^{-1}\right)
$$

where $p_{c}=$ pressure in the capillary water (less one atm), $r_{1}, r_{2}=$ principal curvature radii of the interface, $\gamma=$ surface tension [44] $\left(=72\right.$ dynes $/ \mathrm{cm}$ at $\left.25^{\circ} \mathrm{C}\right)$. The boundary equilibrium condition requires that this surface be tangential to the adsorbed film at the solid surface. Capillary water cannot exist for about $h<0.45$ when $p_{c}$ would exceed the cohesive forces between water molecules $(\approx-1100 \mathrm{~atm})$.

\subsection{Desorption-sorption isotherms. Aging and equivalent curing period}

The total mass of water per unit volume of porous material, $w$, is composed of the adsorbed water $w_{a}$, capillary water $w_{c}$, non-evaporable water $w_{n}$ (which is chemically combined) and a negligible mass of vapor. The dependence of $w$ on humidity $h$ at a given temperature $T$ is a function of the internal geometry of pores which is so complex that empirical relationships must be used. These are called desorption or sorption isotherms $[34,89]$. From the macroscopic viewpoint they represent a material property. An example of their form is given on figure 2. The isotherm for sorption is not identical to the isotherm for the preceding desorption. This irreversibility is caused mainly by the fact that in a pore of given geometry the surface menisci of capillary water may take on various equilibrium forms (" bottle neck" effect and " open-pore " effect [45]). One such possibility is illustrated on figure $3 a, b$, another one on figure $3 c$ showing that at $h=1$ and $p_{a}=0$ the pores need not be full of liquid water but a surface with opposite curvatures, $r_{1}=-r_{2}$, can exist in equilibrium [46]. Irreversibility below $h=0.45$ may be explained by closure of the thinnest gaps on drying (formation of a chemical bond), preventing reentry of adsorbed films [47] or by a similar effect for the evaporable interlayer hydrate which is chemically bound (Appendix C8).

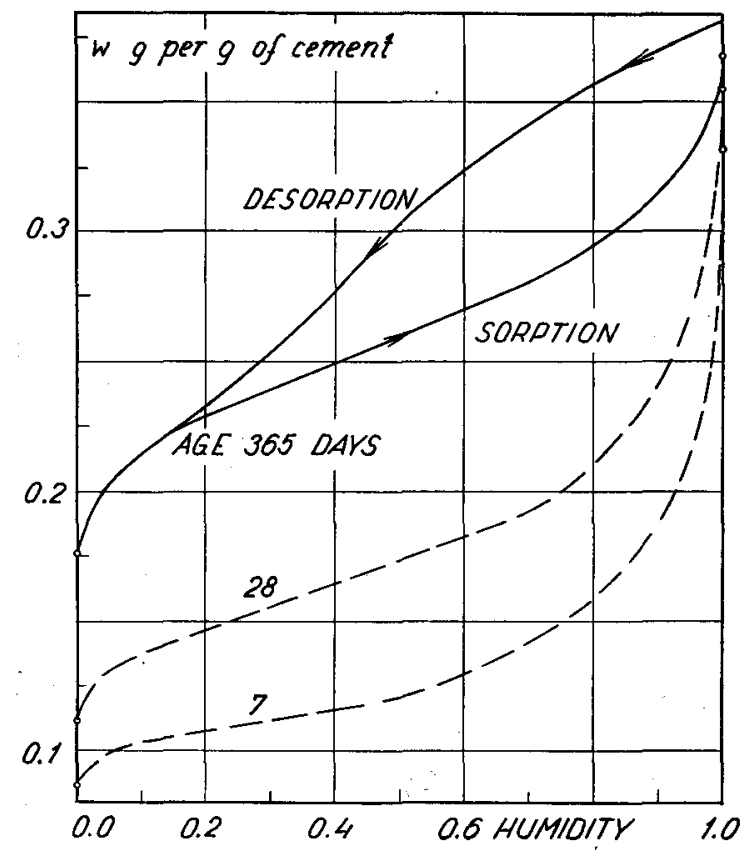

Fig. 2. - Example of desorption-sorption isotherms of cement paste, established by Powers [34].
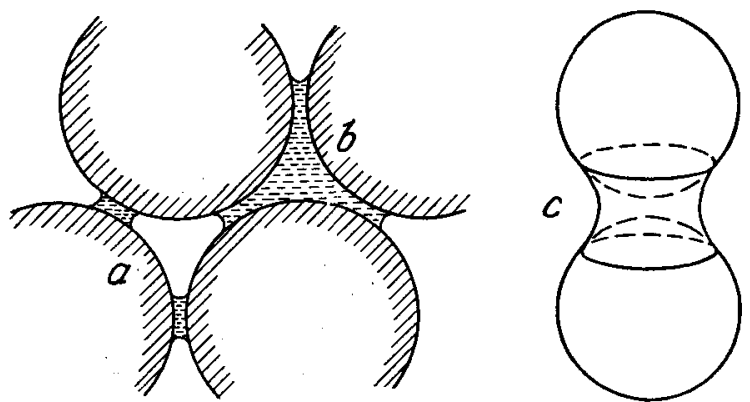

Fig. 3. - Examples of different equilibrium shapes of capillary menisci $(a, b$ for same humidity, $h<1$; $c$ for $h=1[46])$. 
For the desorption or sorption isotherms we can write

$$
d h=k(h) v_{c} d w \text { or } d h=k^{\prime}(h) v_{c} d w \text { where } k v_{c}=(\partial h / \partial w)_{T, t}
$$

where the coefficient $k$ applies for the first desorption and $k^{\prime}$ for the subsequent sorption. Approximately $k$ and $k^{\prime}$ can be considered constant between about 0.1 and 0.8 . $k$ will be called sorption resistance and $k^{\prime}$ desorption resistance (whereas the inverse $1 / k$ may be called sorptivity). Eq. (3) is valid only for a fixed age of cement paste. $v_{c}$ was introduced for convenience as a dimensional constant.

The age, however, is not an objective measure of the maturity or degree of hydration of cement paste. Rather it is the mass of hydrated cement, or the amount of water combined in the solid, $w_{n}$. The increase $d w_{n}$ during the time interval $d t$, characterizing the rate of chemical reaction, is a function of the state variables $h$ (and T) and $w_{n}$. Thus $d w_{n}=\beta_{\mathrm{T}}(h) d w_{n}^{1}$ where $d w_{n}^{1}=f\left(w_{n}\right) d t=$ change of $w_{n}$ at $h=1$ (and reference temperature $\mathrm{T}_{0}$ ) during the time interval $d t ; \beta_{\mathrm{T}}=$ function of $h$ (and $\mathrm{T}$ ) which will be called relative hydration rate. Writing $d w_{n}=f\left(w_{n}\right)\left[\beta_{\mathrm{T}}(h) d t\right]$, we are led to the following definition (see Fig. 4) :
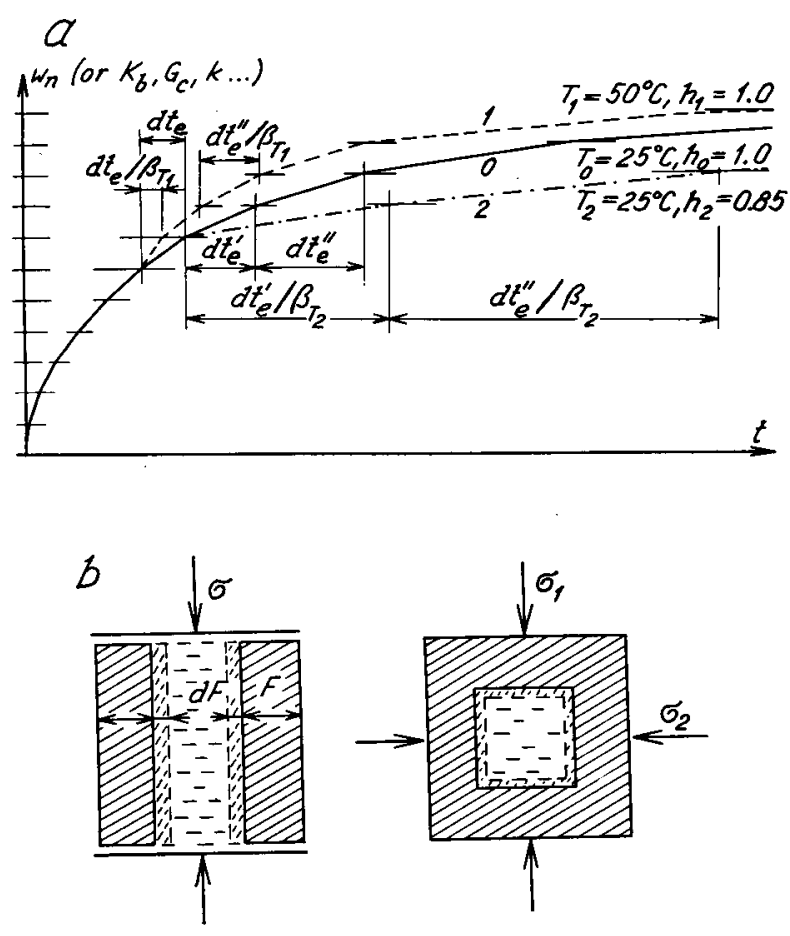

Fig 4. -- Schemes for interpretation of the effect of aging,

a) definition of the equivalent curing period at variable $T$ and $h$,

b) change of specific mass of porous material.

$$
d t_{e}=\beta_{T}(h) d t \text { or } t_{e}=\int_{0}^{t} \beta_{T} d t^{\prime}
$$

$t_{e}$ will be called equivalent curing period. $t_{e}$ represents the period of curing at $h=1$ and reference temperature $T_{o}$ at which the same amount $w_{n}$ would become combined as for a given time-variable $h$ (and T) in time $t$. Approximately, we can probably consider $\beta_{\mathrm{T}}$ (not $d w_{n}$ ) as independent of $w_{n}$, or $t_{e}$. For $h=1$ and $\mathrm{T}=\mathrm{T}_{0}, \beta_{\mathrm{T}}=1$. Below a certain humidity, equal to about 0.8 (as determined by self-desiccation of sealed samples with unsufficient water-cement ratios [46]), $\beta_{\mathrm{T}}=0$, and even up to $h=0.95$ probably $[48] \beta_{\mathrm{T}} \ll 1$. of $t_{e}$

All material parameters, e.g. $k$ (or $\mathrm{K}_{b}, \mathrm{~K}_{c}, \mathrm{G}_{c}, \mathrm{G}_{d}, f_{d}, f_{a}, d, \varphi_{d}, \varphi, c, x, \ldots$ defined later), are functions

Effect of hydration on sorption isotherms is seen in figure 2 . Since in a time interval $d t$ the part $d w_{e}$ of the evaporable water $w_{a}+w_{c}$ is lost, becoming combined in the solid, i.e. $d w_{n}=-d w_{e}$, the humidity is decreased by a certain value $d h_{s}$. However, $d h_{s}$ is much less than $-k d w_{n}$ since the porosity becomes also decreased (because cement doubles its volume at hydration [49]). Moreover, the value of $k$ for $h \rightarrow 1$ is rather uncertain and possibly very small (Fig. 2). Therefore, it is not suitable to use the function $w_{n}\left(t_{e}\right)$ for calculations of humidity [50]. We shall rather introduce directly the function $h_{s}\left(t_{e}\right)$ representing the so-called self-desiccation of sealed samples $[51,46]$, a directly measurable quantity. Thus at variable age we have

$$
d h=k\left(h, t_{e}\right) v_{c} d w+d h_{s}\left(t_{e}\right)
$$

For normal water-cement ratios (greater than 0.5 ) there is in cement paste $[51] h_{s}(\infty) \geqslant 0.95$ (for suitable expression for $h_{s}$ see eq. 60). 


\section{BASIC ASSUMPTIONS FOR THE STRESS AND STRAIN ANALYSIS}

The facts about the cement paste structure (see Introduction) justify the following assumption.

\section{Assumption 1.}

The porosity is great and internal surface area is large. This implies that a large part of the evaporable water is in the state of hindered adsorption [32-34]. Consequently, the number of hindered adsorbed layers intersecting a unit length is great and their total thickness is not small with respect to unity.

\section{Assumption 2.}

Furthermore, we require that the deformations, their rates and water flow rates are small, so that the stress-strain law be linear. It has been proved experimentally $[2-4,8,19,20,50,12,52]$ that this linearity is approximately acceptable for concrete for stresses less than about 0.4 of its strength, except for unloading and repeated stresses.

\section{Assumption 3.}

The material is macroscopically isotropic.

The smallness of water flow rate allows us to assume that the thermodynamic equilibrium at the interface of various phases of water, expressed by eqs. (1) and $(2 a)$, is maintained at any time (especially in each pore of capillary size). Eq. (2) is an equilibrium condition which is to be achieved asymptotically at the end of the creep process. Because adsorption has a dynamic character [41], water molecules can migrate or flow along the adsorbed layers. This flow may occur even without leaving the adsorbed layer [54] so that diffusion along the hindered layers is also possible. Thus, with respect to eq. (2), the difference $p_{d}-p_{a}$ causes a local diffusion between the hindered and unhindered adsorbed layers.

Our assumptions imply that the average relative change of thickness (with respect to the thickness) of hindered adsorbed layers, accompanying deformation, is always small. The total volume of water which may be "squeezed out" of (or "imbibed into") all the hindered layers in a unit volume of material cannot be greater than the relative volume change of material, and therefore must be negligible in comparison with the volume of pores even if the load-bearing water is subtracted. This small volume added to (or removed from) the non-bearing evaporable water in pores increases (or decreases) the humidity. However, this increase must be negligible since according to the slope of the sorption isotherm (Fig. 2) a small change of water content produces a small change of humidity. This fact has an important mathematical consequence-the macroscopic water flow problem is uncoupled with the stress and strain problem and may be solved independently (unlike in the vibration of the saturated sand [39]).

Note. This result is also supported directly by the fact that the load does not produce an appreciable change of water content and humidity during creep [32]. This does not contradict the fact that the loss of water needed to produce shrinkage equal to creep is great (250 times greater [32]). The difference of creep between sealed and unsealed samples [36] cannot be explained by a rise in humidity caused by load in a sealed sample. (It is rather explained by the term with $h$ in eq. (25), (37)).

For the mathematical treatment we have to idealize the microstructure. We imagine the material to be composed of two constituents, the fluid and the solid framework. The latter includes hydrated cement crystals and unhydrated cement grains. The thin hindered adsorbed layers form also a part of the solid framework since their immediate response depends only on the deformation of the solid, while their delayed response depends on the diffusion along the hindered adsorbed layers into larger pores. The fluid component, the state of which is independent of deformation, includes water vapor, capillary water and free adsorbed layers, that is, all water contained in voids of capillary size.

Note. A sharp distinction, however, cannot be made. For instance, with the decrease of humidity some hindered layers become unhindered. Moreover, e.g. at volume compression, the change of pressure $p_{d}$ in various layers is statistically different, and this difference also causes a local flow between various hindered layers.

In an exact approach we should have to assume the statistical concept of the geometry of microstructure. Its complexity, however, compels us to work, more or less intuitively, only with certain " average" quantities. Especially, we have to realize the difference between the macroscale and microscale and distinguish properly between the macroscopic diffusion, representing the average movement of water molecules, and the microscopic local diffusion which is characterized by zero average movement (in a region sufficiently large with respect to particle size but sufficiently small with respect to body dimensions). The local diffusion (resulting from thermodynamic disequilibrium between adjacent free and hindered adsorbed layers) appears in the macroscale as a material property and must thus be expressed in the macroscopic constitutive equation. 


\section{MACROSCOPIC DIFFUSION OF WATER AND ANALYSIS OF HUMIDITY}

In a uni-dimensional flow the change $d w$ of the mass $w$ of water in a unit volume element during the time interval $d t$ is

$$
v_{c} d w=-\frac{\partial \dot{u}}{\partial x} d t
$$

where $u$ is the average displacement of water molecules, $\dot{u}=\partial u / \partial t . v_{c}=1 \mathrm{~cm}^{3} / \mathrm{g}$ ).

Considering that the mean free path of water vapor molecules at $25{ }^{\circ} \mathrm{C}$ and 1 atm is about $800 \AA$ while the average pore size in cement paste is about $15 \AA$ (and that the capillaries are discontinuous [53]), we may conclude that the flow of adsorbed water molecules along the layers, called surface diffusion [54], is much more significant than the flow of vapor (evaporation-condensation theory [55]) or capillary water, despite much higher (perhaps $10^{5}$ times) "viscosity". Therefore, the average speed of flow of water molecules is governed essentially by the gradient of $p_{a}$ rather than $p_{v}$, and we can write

$$
\dot{u}=-\sigma_{T} c(h) \frac{\partial p_{a}}{\partial x}
$$

where $b_{\mathrm{T}} \mathrm{C}$ represents permeability. For simplicity we assume that $b_{\mathrm{T}}$ depends only on $\mathrm{T}$, and $\mathrm{C}$ only on $h$. According to (1) we can write as well

$$
\dot{u}=-b_{T} c(h) \frac{\partial h}{\partial x} \quad \text { where } c(h)=R T C(h) /\left(M_{a} h\right)
$$

Substitution into (6) yields

$$
v_{c} \frac{\partial w}{\partial t}=\frac{\partial}{\partial x}\left[b_{r} c(h) \frac{\partial h}{\partial x}\right]
$$

Using the sorption isotherm (4) we can eliminate $w$ and we get finally the following nonlinear partial differential equation for $h$,

$$
\frac{\partial h}{\partial t}-\frac{\partial h_{s}}{\partial t}=k(h) \frac{\partial}{\partial x}\left[b, c(h) \frac{\partial h}{\partial x}\right]
$$

Note. For two-dimensional flow we should have to add at the right hand side another term of the same form, with $y$ instead of $x$.

By analysis of some concrete drying tests [56, 57, 12] we can find that $b_{\mathrm{T}} c h \approx 0.1 \mathrm{~cm}^{2} / \mathrm{day}$ at $h \approx 0.7, \mathrm{~T}=25^{\circ} \mathrm{C}[56]$ and $2 \mathrm{~cm}^{2}$; day at $93^{\circ} \mathrm{C}$ [57] (for those particular concretes used).

At the surface of the body the amount of water coming from inside must be equal to the amount of water evaporated (from adsorbed state) into the environment. Let $x$ denote the outward normal of the surface. Then, assuming that the rate of evaporation is proportional to the difference in partial free energies (Appendix A) (at the same temperature), we can write

$$
\dot{u}=-B\left(\ln h_{e x}-\ln h\right)
$$

where $h_{e x}=$ humidity of the external environment, $\mathrm{B}=$ coefficient of water transmission at the surface depending on humidity $h$ and temperature T. By substitution of expression (7b) we get the boundary condition

$$
b_{T} c(h) \frac{\partial h}{\partial x}+B \ln \frac{h}{h_{e x}}=0
$$

Often the rate of evaporation is much faster than the rate of humidity change inside the specimen. Then we can let $B \rightarrow \infty$ and get

$$
h \approx h_{e x}
$$

If the surface is perfectly sealed, we have $B=0$ or

$$
\partial h / \partial x=0
$$

Eqs. (8) and (9) formulate the boundary value problem of water diffusion, from which the humidity $h$ as a function of $x, y, z, t$ may be solved. This problem is nonlinear. 
The diffusion coefficient $c(h)$ must decrease strongly with the thickness of the adsorption layer, as the attraction forces decrease sharply with the distance from the surface [41]. This holds true even if the average thickness of the layer is less than one molecule [54]. We can thus introduce

$$
C(h) \sim \delta_{\alpha}^{n+1}
$$

and because approximately $\delta_{a} \sim h$ we can write $\mathrm{C}(h)=h^{n+1}$ or

$$
c(h) \approx h^{n}
$$

If we were to assume that this effect is similar to a linearly viscous fluid flowing in a gap between two planes of thickness $2 \delta_{a}, \dot{u}$ vould be distributed parabolically ower the thickness and $\dot{u}$ would be proportional to $\delta_{a}^{2}$ so that we would have $n=1$. Because of the rapid decrease of the surface attractive forces with the distance it is more realistic to assume $n>1$. On the other hand, in a hindered adsorbed layer the thickness is independent of $h$, so that $\mathrm{C}=$ const. or $n=-1$. This latter case seems, however, to be of minor importance as the macroscopic diffusion tends to pass mostly through the regions of minor resistance, i.e. the thicker unhindered layers, provided that some of them are continuous. Another reason for higher $c$ in a hindered layer is that the molecules cannot temporarily leave the layer during migration. For the vapor diffusion alone, $\mathrm{C} \approx$ const. or $c \sim 1 / \mathrm{h}$. Thus, unlike for diffusion in adsorbed layers, $c$ does not decrease with $h$ but it increases. Hence vapor diffusion may become dominant for $h \rightarrow 0$. An expression allowing for a combined effect is $\mathrm{C}(h)=\mathrm{C}_{1}+\mathrm{C}_{2} \delta_{a}^{n+1}$ or $c(h)=c_{1} / h+c_{2} h^{n}$ where $\mathrm{C}_{1}, \mathrm{C}_{2}, c_{1}, c_{2}$ are constants (and $c_{1} / 0.5 \ll c_{2} \times 0.5^{n}$ ).

Note. The variability of $c(h)$, eq. (10), explains why the core of massive concrete bodies exposed to drying retains a high humidity for a much longer period than linear diffusion would predict. The reason is that the conductivity of the surface region drops as it dries up.

For $h \rightarrow 1$ there seems to be a continuous transition to the case of flow in saturated cement paste under hydrostatic pressure $p$ (in excess of $1 \mathrm{~atm}$ ). Because in eq. (10) we assume $c=1$ for $h=1$, eq. (7a) must be replaced (at $25^{\circ} \mathrm{C}$ ) by

$$
\dot{u}=-\frac{M v_{Q}}{R} \frac{b_{T}}{T} \frac{\partial p}{\partial x}=-\frac{\sigma_{T}}{1360} \frac{\partial p}{\partial x}
$$

where $b_{\mathrm{T}} / 1360$ represents permeability $[58,59]$.

Note. The significance of eq. (11) may be demonstrated with the case of a retaining wall of thickness $l$, one face of which is immersed in water of hydrostatic overpressure $p_{1}$ and the other face exposed to atmosphere of humidity $h_{2}$. Because in a steady state the values of $\ddot{u}$ following from eqs. (11) or (7b) must be constant over the thickness of wall, the condition $c(h) \partial h / \partial x=(\partial p / \partial x) / 1360$ must be fulfilled. Consequently, the distance of the point at which $p=0$ and $h=1\left(p_{a}=0\right)$ from the immersed face is found to be equal to

$$
p_{1} l\left[p_{1}+1360\left(1-h_{2}^{n+1}\right) /(n+1)\right]^{-1}
$$

Thus for $p_{1}=10 \mathrm{~atm}, h_{2}=0.5, n=2$, this distance is found to be only $0.0227 l$. This enormous infuence of the gradient of the adsorbed water pressure has often been disregarded.

However, if concrete has a system of microcracks (mostly bond cracks) the water will tend to pass through these passages of minor resistance when they are full of water $(h=1)$. Thus the effective value of $b_{\mathrm{T}}$ will increase discontinuously at $h=1$, and much deeper penetration of water into the wall would be obtained. Especially, after a cycle of drying, microcracks may be created, increasing enormously the permeability (about 70 times [59]), although the deformation properties, depending mainly on the strongest parts of the structure, might be changed only slightly.

\section{MICROSCOPIC LOCAL DIFFUSION AND CONTRACTION OF THE HINDERED ADSORBED LAYERS}

Let us examine now the typical configuration as shown in figure 1. Let the average pressure in the hindered layer be designated by $\bar{p}_{d}$ and the volume stress in load-bearing water be defined as the resultant of $p_{d}$ per unit area of the porous material, i.e.

$$
\sigma_{d}=-f_{d} \bar{p}_{d}
$$


where $f_{d}$ is the effective area factor for the load-bearing water. $f_{d}$ decreases with a decreasing amount of water adsorbed, $w_{a}$, and for $h \rightarrow 0$ tends to zero; always $f_{d}<1$. Most simply we can assume [32] :

$$
f_{d}=f_{d}(h) \sim h
$$

With regard to the irreversibility of desorption isotherms, $f_{d}$ is smaller for sorption than for desorption. The pressure $p_{d}$ is linked with the specific volume as follows

$$
d v_{d}=-\beta_{a} d p_{d}
$$

where $\beta_{a}=$ volume compressibility of the adsorbed water $=v_{a}{ }^{-1}\left(\partial v_{a} / \partial p_{a}\right)_{\mathrm{T}}$. Its value might be rather different from $\beta_{w}$ for the liquid water which is $\beta_{w}^{-1}=25,000 \mathrm{kp} / \mathrm{cm}^{2}$ while for cement gel [60] $\beta_{c}^{-1}=240,000 \mathrm{kp} / \mathrm{cm}^{2}$.

A change of the pressure $p_{d}$ in the hindered adsorbed layer, caused by compression of the material or by a change of humidity in larger pores, violates the condition (2) of thermodynamic equilibrium with the non-bearing water. Hence a flow of water out of (or into) the hindered layer results. Let us define $\bar{w}_{d}$ as the average total mass per unit area of all load-bearing layers intersecting a unit length. Their average total thickness $\bar{\delta}_{d}$ is then expressed as $\bar{\delta}_{d}=\bar{v}_{d} \bar{w}_{d}$. From this is follows that

$$
\begin{aligned}
d \bar{\delta}_{d} & =\bar{v}_{d} d \bar{w}_{d}+\bar{w}_{d} d \bar{v}_{d} \\
\text { or } \quad d \bar{\delta}_{d} & =v_{a} d \bar{w}_{d}-\frac{\bar{\delta}_{d}}{v_{a}} \beta_{a} d \bar{p}_{d}
\end{aligned}
$$

where $v_{a} \approx \bar{v}_{d}$ and $\bar{\delta}_{d}$ may be considered as constants according to assumptions 1 and 2 . The total amount of water which has to diffuse along the hindered adsorbed layer out to the adjacent capillary pore is $f d w_{d}$. Introducing the idea of average effective distance $\bar{d}=\bar{d}(h)$ between load-bearing water and the adjacent non-bearing water and keeping in mind the equilibrium condition (2), we can write for this diffusion (to the first order)

$$
f_{d} d \bar{w}_{d}=-a_{T} \frac{\bar{p}_{d}-p_{a}}{\bar{d}(h)} d t
$$

where $a_{\mathrm{T}}$ is a diffusion constant at a given temperature T. Substituting for $\overline{d \bar{w}_{d}}$ and $\bar{p}_{d}$ from eqs. (15) and (12) we get, after rearrangements,

$$
\frac{\partial \bar{\delta}_{d}}{\partial t}=\frac{1}{K_{d}} \frac{\partial \sigma_{d}}{\partial t}+\varphi_{d}\left(\sigma_{d}-\sigma_{a d}\right)-\frac{\partial h}{\partial t} \frac{\sigma_{d}}{K_{f}} \text { (17) }
$$

where the following notation has been introduced

$$
\begin{aligned}
& K_{d}=v_{a} f_{d} /\left(\beta_{a} \bar{\delta}_{d}\right), \quad K_{f}=K_{d} f_{d}\left(d f_{d} / d h\right)^{-1}(18) \\
& \varphi_{d}=v_{a} a_{T} /\left(f_{d}^{2} \bar{d}\right)
\end{aligned}
$$

We also defined $\sigma_{a d}=-f_{d} p_{a}$. $\sigma_{a d}$ represents the theoretical value of the stress $\sigma_{d}$ in load-bearing water which is needed for thermodynamic equilibrium at a given humidity $h$.

With $\sigma_{a d}=-f_{a} p_{a}$, however, for an unloaded specimen, immersed in water since the time of casting, no volume change would be obtained. Actually, there will be swelling, though relatively small $[2,15]$, for other reasons, specifically not considered. Phenomenologically and most simply, eq. (17), may be adjusted to account for this if we put

$$
\sigma_{a d}=-f_{d}\left[p_{a}(h)-p_{a}\left(h_{0}\right)\right]=\frac{R T}{M v_{a}} f_{d} \ln \frac{h_{0}}{h}
$$

and define a decrease of humidity $h_{o}$ as such a function of $t_{e}$, for which the volume change is just zero. For concretes for dams probably $h_{o}\left(t_{e}\right) \doteq h_{s}\left(t_{e}\right)$ since the autogenous shrinkage (see Appendix C14) is practically zero $[15,61]$.

Note. It is true that water is imbibed [2] into a specimen stored under water, due to osmotic pressure, self-desiccation and hydraulic over-pressure, but our expressions for $p_{a}$ and $\sigma_{a d}$ at $h=1$ are still same. To be exact, $p_{\text {sat }}$ in eq. (1) should be made dependent 
on the concentration of the aqueous solution which varies with the progress of hydration (as well as $w$ ), and causes osmotic pressure with respect to the relatively pure water outside the specimen. Major cause of swelling, however, is probably the growth in volume of cement gel (see Appendix C9).

With the simple assumption $f_{d} \sim h, \sigma_{a d}$ tends to infinity for $h \rightarrow 0$. Actually, this observation makes no sense since eq. (1) is not valid for $h \rightarrow 0, v_{a}$ being variable for $h \rightarrow 0$. A suitable expression for $\sigma_{a d}$ will be given later in eq. $(60)$.

\section{CONSTITUTIVE EQUATION AT VARIABLE HUMIDITY}

\subsection{Concrete as a porous material of variable mass of the solid component}

The increase of the elastic modulus of cement paste and concrete with age cannot be caused by a change of the modulus $K_{c}$ of the hydrated cement itself, but rather by the increase of its mass per unit volume of porous material which is due to continued hydration [49], i.e., conversion of anhydrous cement to hydrated cement. In general, for an elastic material two possibilities exist for taking this into account, depending on whether the removed or added matter is in a stressed state or an unstressed state. The first case occurs if a body under stress is being dissolved, with the elastic energy of the removed matter being transformed into heat. Then (Fig. 4) $d \sigma=\mathrm{FK}_{c} d \varepsilon+\mathrm{K}_{c} \varepsilon d \mathrm{~F}$ or

$$
\sigma(t)=F(t) K_{c} \varepsilon(t)=K(t) \varepsilon(t)
$$

where $\sigma, \varepsilon$ are the average stress and strain in the porous material, $F$ the effective area of solid per unit area, $K$ the effective modulus of the porous material. If the matter is being added by a chemical process, this is always done in an unstressed state. Thus

$$
d \sigma(t)=F(t) K_{c} d \varepsilon(t)=K(t) d \varepsilon(t)
$$

Clearly eq. (21), introduced in 1965 [22, 23], is not equivalent to the first relationship, which had been used incorrectly in the past in rheological models for concrete. In order to account for various humidity conditions, $t$ must be replaced by $t_{e}$, as an independent variable for $K$.

Having formulated the behavior of both the elastic and fluid components of our porous material, we are ready to study the composite.

\subsection{Volumetric creep and shrinkage}

For deformations and deformation rates sufficiently small the response of any material to stresses and strains may be considered linear. From the general theory of continuous media [62, 63] it is known that the deviatoric and volumetric behaviour are separable if, and only if, the stress-strain law is linear and the material isotropic. First we shall investigate the volumetric behaviour. (For higher stresses this separability is not true [98]).

Let us denote by $\sigma$ the average total volume stress per unit area of the porous material, $\sigma=\left(\sigma_{11}+\sigma_{22}+\sigma_{33}\right) / 3$. $\sigma$ may be decomposed into the stress in the fluid, $\sigma_{a}$, representing the resultant of the pressure $p_{a}$ in the capillary water and unhindered adsorbed layers per unit area of the porous material, and the stress in the solid framework which equals $\sigma-\sigma_{a}$ according to the static equilibrium condition in the two-phase material. $\sigma-\sigma_{a}$ is the resultant of the stresses in the hydrated cement, the hindered adsorbed layers between them and the unhydrated cement grains. simply

It is expedient to express $\sigma_{a}=-f_{a} p_{a}$ where $f_{a}=$ area factor for the fluid. Then we can write

$$
\sigma_{a}=\sigma_{a d} f_{a} / f_{d}
$$

According to our assumptions, $\sigma_{a}$ is independent of deformation (unlike in a medium with pores saturated by liquid, at high flow rates [39]). The provenance and significance of the terms $\sigma_{a}$ and $\sigma_{a d}$ is different. $\sigma_{a}$ is needed for the static equilibrium while $\sigma_{a d}$ arises from the thermodynamic equilibrium condition.

Note. The stress in the fluid alone, $\sigma_{a}$, does not satisfy the differential equilibrium conditions. These must be imposed upon the total stress $\sigma$ (plus $s_{i j}$ ). The constitutive equation for the fluid is represented by eq. (5).

The average total volume strain will be designated by $\varepsilon, \varepsilon=\left(\varepsilon_{11}+\varepsilon_{22}+\varepsilon_{33}\right) / 3$. It is composed of (a) shrinkage $\varepsilon^{s}$, defined as the deformation for $\sigma=0$ and $d T=0,(b)$ thermal dilatation $\varepsilon^{\mathrm{T}}$, defined by the condition $\sigma=0$ after subtraction of shrinkage, and $(c)$ creep $\varepsilon^{c}$ which represents the rest and is caused by the stress $\sigma . \quad \varepsilon^{c}$ includes the instantaneous deformation, which is the limit for the delay of response tending to zero. 
In eq. (17) for the hindered layers, the stress $\sigma_{d}$ and the thickness $\bar{\delta}_{d}$ are not known, as they result from the interaction with the elastic parts of the solid framework, modelled by springs obeying eq. (21). The simplest model possible for this interaction within the solid framework is the three-element model in figure 5a. It consists of a spring and a unit formed by a spring coupled in parallel with the "sorption element" [36], described by eq. (17). Later we shall refer to this unit as a Powers' unit since it was first conceived and verbally discussed by Powers [36]. We imagine that the extension of the model corresponds to $\varepsilon$. Its load is $\sigma-\sigma_{a}$.

The stress $\sigma_{c}$ carried by the spring $K_{c}$ in figure $5 a$ equals $\sigma-\sigma_{a}-\sigma_{\text {disj }}$ where $\sigma_{\text {disj }}$ is the resultant of the disjoining stresses $p_{\text {disj }}$ that are developed by the hindered layer on its solid surface. By definition (see also eq. A5), $p_{\text {disj }}=p_{d}-\left[p_{a}\right]_{\delta_{d}}$ Hence

$$
\sigma_{d i s j}=-\int_{f_{d}} p_{d i s j} d f_{d}=\sigma_{d}-\sigma_{a}^{d i s j}
$$

where $\sigma_{a}^{\text {disj }}=-\int_{f_{d}}\left[p_{a}\right]_{\delta_{d}} d f_{d}=$ function of $h$ and $\mathrm{T}$ (and $t_{e}$ ). Consequently, $\sigma_{c}=\sigma-\sigma_{a}^{\prime}-\sigma_{a}$ where $\sigma_{a}^{\prime}=\sigma_{a}-\sigma_{a}^{\text {disj. }}$.
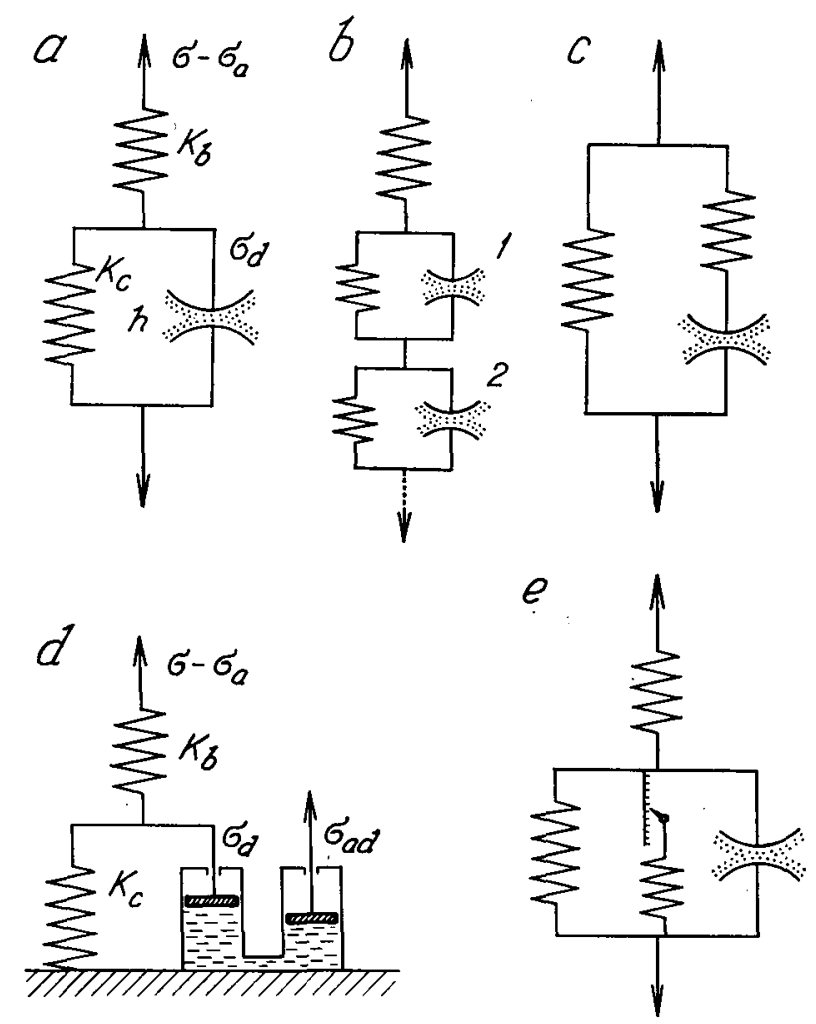

FIG. 5. - Rheological models for interaction of the elastic particles of cement paste or concrete and the hindered layers. $a, b, c, e$ models with sorption elements, $d$ model equivalent to $a$, with two pistons interconnected by a thin tube of high viscous resistance (for $\sigma_{a d}=\mathbf{0}$ the pistons may be replaced by a dashpot), $e$ model with a " ratchet" for irreversible deformation.

Eq. (20) for the two springs in figure 5 a may now be written in the following form (using the notation $\dot{\varepsilon}=\partial \varepsilon / \partial t$, etc.):

$$
\begin{aligned}
& \dot{\varepsilon}-\dot{\varepsilon}_{d}=\left(\dot{\sigma}-\dot{\sigma}_{a}\right) / K_{b}\left(t_{e}\right) \\
& \dot{\varepsilon}_{d}=\left(\dot{\sigma}-\dot{\sigma}_{a}^{\prime}-\dot{\sigma}_{d}\right) / K_{c}\left(t_{e}\right)
\end{aligned}
$$

where $d \varepsilon_{d}=d \bar{\delta}_{d} . K_{b}, K_{c}$ are the elastic constants of the springs; they are increasing functions of $t_{e}$, $\mathbf{K}_{b}=\mathbf{K}_{b}\left(t_{e}\right), \mathbf{K}_{c}=\mathbf{K}_{c}\left(t_{e}\right)$. 
By substitution of eq. (17) for $d \varepsilon_{d}=d \bar{\delta}_{d}$ and further algebraic rearrangment we obtain:

$$
\begin{aligned}
& \dot{\varepsilon}=\frac{\dot{\sigma}-\dot{\sigma}_{a}^{\prime}-\dot{\sigma}_{a}^{\prime \prime}}{K}+\frac{\varphi}{K_{c}}\left(\sigma_{d}-\sigma_{a d}\right)-\frac{\dot{h}}{K_{h}} \sigma_{d} \\
& \frac{\dot{\sigma}_{d}}{K_{c}}=\frac{\dot{\sigma}-\dot{\sigma}_{a}^{\prime}}{K_{a}}-\dot{\varepsilon}-\frac{\dot{\sigma}_{a}^{\prime \prime}}{K} \\
& \left.\begin{array}{l}
k_{a}=\left(k_{b}^{-1}+k_{c}^{-1}\right)^{-1}, \quad k_{h}=k_{f}\left(1+k_{c} / k_{d}\right) \\
k=\left[k_{b}^{-1}+\left(k_{c}+k_{d}\right)^{-1}\right]^{-1}
\end{array}\right\} \\
& \varphi=\varphi_{d} k_{c} /\left(1+k_{c} / K_{d}\right)
\end{aligned}
$$

In addition, the notation $\dot{\sigma}_{a}^{\prime \prime}=\dot{\sigma}_{a}^{\text {disj }} \mathbf{K} / \mathbf{K}_{b}$ has been also introduced.

In the rest of this paper, we shall assume for simplicity that:

$$
\dot{\sigma}_{a}^{\prime \prime} \doteq 0, \quad \dot{\sigma}_{a}^{\prime} \doteq \dot{\sigma}_{a}
$$

Eqs. (25) and (26) represent the volumetric constitutive equation or stress-strain law.

Note. We write partial derivatives $\dot{\sigma}=\partial / \partial t \ldots$ because $\sigma, \sigma_{d}, \varepsilon, \ldots$ etc. are also functions of the spatial coordinates $x, y, z$. Since the deformations are small the so-called objective material derivatives [62] are not needed.

Let us now discuss the coefficients (27) and (28). K represents the instantaneous volume modulus. $K_{d}$ accounts for that part of $K$ which depends on the moisture content. Since $K$ is known to decrease only slightly with drying $[28,12], K_{d}$ must be rather high with respect to $K_{c}$ (see eq. 60). Approximately, we may let $K_{d} \rightarrow \infty$, and then $K \doteq K_{b}$. Therefore the instantaneous elastic response is essentially reflected by $K_{b} . \quad K_{d}$ corresponds to the volume compressibility of the adsorbed water in hindered layers. The direct action of van der Waals forces between the solid surfaces across the hindered layer is represented by $K_{b}$ and $K_{c}$, along with the elastic deformation of hydrated cement particles and unhydrated grains. In the case of no aging, $K_{a}$ represents the effective modulus for the "final " deformation as $t \rightarrow \infty$.

The form of dependence of $\varphi$ upon $h$ may be guessed with the help of some geometric hypotheses about the form of the thin gaps between particles. This is done in Appendix B. The results suggest that probably $\varphi \sim h$ or $h /(1+h)$. This expression agrees with the fact that the creep of dried specimens is negligible $[28,31]$, and that creep is the greater, the higher is $h$ if $h$ is kept time-constant during creep [30], [31], [1], [2], [29].

The term depending on $\dot{h}$, in eq. (25), is positive if $\dot{h}<0$ as in the case of drying environment. It thus probably accounts for the increased creep at drying, i.e. "drying creep " [16]. In our derivation the origin of this term was obscured. It arose because in eq. (15) we had to substitute, with respect to (12), $d \bar{p}_{d}=\left(-d \sigma_{d}+\bar{p}_{d} d f_{d}\right) / f_{d}$, and not $d \bar{p}_{d}=-d \sigma_{d} / f_{d}$. The second term $\bar{p}_{d} d f_{d}$ accounts for the fact that $\bar{p}_{d}$ is increased if $f_{d}$ is decreased and $\sigma_{d}$ kept constant. Decreasing $h$, we not only diminish $p_{a}$ in the difference $\bar{p}_{d}-p_{a}$, but we also decrease $f_{d}$ and thus increase $\bar{p}_{d}$ even if $\sigma_{d}$ remains unchanged. Therefore, drying conditions cause the driving force of microscopic diffusion, $\bar{p}_{d}-p_{a}$ to be amplified in comparison with stabilized humidity conditions. We have thus an explanation of the seemingly confusing effect of humidity, i.e. smaller creep for lower $h$ at $\dot{h}=0$ on one hand, and increase of creep at $\dot{h} \ll 0$ on the other hand.

For the wetting process, $\dot{h}<0$, the water in the extended part of the hindered layer (corresponding to $d f_{d}$ ) is not under pressure $\overline{p_{d}}$ but rather $p_{a}$.)

Therefore the above-mentioned term $\bar{p}_{d} d f_{d}$ is replaced by $p_{a} d f_{d}$ and the effect described above does not occur. For this reason $k_{h}$ is probably much higher for $\dot{h}>0$ than for $\dot{h}<0$. (A more relevant analysis of $k_{h}$ could be made as in Appendix B, considering variable $x_{a}$ and compressible fluid).

It can be verified that eqs. (25), (26) might also be formally interpreted by the model in figure $5 d$, containing two pistons connected by an orifice of variable size instead of a sorption element. Furthermore it should be mentioned that another possibility for a three-element model exists, as seen on figure $5 c$. It is not completely equivalent to figure $5 a$ [22].

For a prescribed $\sigma(t)$ [or $\varepsilon(t)]$ eqs. (25) and (26) represent a system of two ordinary linear differential equations in $\varepsilon(t)$ [or in $\sigma(t)]$, with variable coefficients. They can be reduced to one second order equation, eliminating $\sigma_{d}$. For this purpose eq. (25) is multiplied by $\mathbf{K}_{c} / \tilde{\varphi}$ where

$$
\widetilde{\varphi}=\varphi-\hbar K_{c} / K_{h}
$$


Then it is differentiated and eq. (26) substituted for $\dot{\sigma}_{d}$. This yields

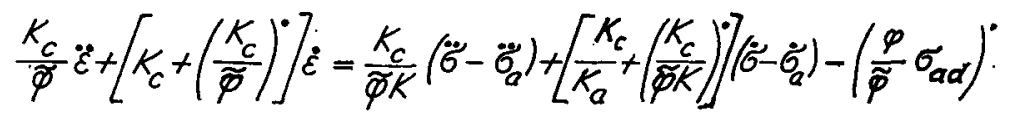

where $\ddot{\varepsilon}=\partial^{2} \varepsilon / \partial t^{2},()^{*}=\partial / \partial t$ etc. Because eq. (25) was differentiated it must be used as the initial condition for this equation.

In old concrete or in the case of sufficiently low humidity the hydration process stops, so that $K_{b}, K_{c}$ and $K_{a}$ become approximately constant. Then eq. (26) yields $\sigma_{d} / K_{c}=\left(\sigma-\sigma_{a}\right) / K_{a}-\varepsilon$. By substitution into (25), instead of eq. (30) we get

$$
\dot{\varepsilon}+\tilde{\varphi} \varepsilon=\frac{\dot{\sigma}-\dot{\sigma}_{a}}{k}+\frac{\tilde{\varphi}}{k_{a}}\left(\sigma-\sigma_{a}\right)-\frac{\varphi}{k_{c}} \sigma_{a d}
$$

For a prescribed $\sigma(t)[$ or $\varepsilon(t)]$, the solution $\varepsilon(t)$ [or $\varepsilon(t)]$ of the system $(25),(26)$ or eq. (30) can be expressed explicitly by quadratures, using the method of variation of constants. For the sake of simplicity we shall demonstrate this only for the special case of eq. (31). It may be verified by back substitution in (43) that its integral is (for $\dot{K}_{d} \approx 0$ )

$$
\begin{aligned}
& \varepsilon(t)=\frac{\sigma(t)}{K}+\int_{0}^{t} \sigma(\tau) L(t, \tau) d \tau+\varepsilon_{0}^{s}(t) \\
& \text { where }\left(\sigma_{a}(0)=0\right) \text { : } \\
& L(t, \tau)=\left[\frac{\tilde{\varphi} / K_{c}}{\left.1+k_{c} / k_{d}\right] \tau}\right]^{-\int_{\tau}^{t}[\tilde{\varphi}]_{\tau^{\prime}} d t^{\prime}} \\
& \varepsilon_{0}^{s}(t)=-\frac{\sigma_{a}(t)}{k}-\int_{0}^{t} \sigma_{a}(\tau) L_{a}(t, \tau) d \tau \\
& \text { where } L_{a}(t, \tau)=L(t, \tau)+\left[\frac{\varphi}{k_{c}} \frac{f_{d}}{f_{a}}\right]_{\tau} e^{-\int_{\tau}^{\tau} \widetilde{\varphi} d t^{\prime}}
\end{aligned}
$$

$\mathrm{L}$ represents the volume stress memory function and $\mathrm{L}_{a}$ may be called the humidity memory function.

Eq. (32) is a formulation of the stress-strain law which is equivalent to eqs. (25), (26) or eq. (30) since these equations may be deduced from it. It has the advantage that its form is the same for any more complex linear model (eqs. $32 e, f$ ). It resembles the creep law of classical viscoelasticity, but in concrete the memory function $\mathrm{L}(t, \tau)$ as well as $\mathrm{L}_{a}(t, \tau)$ is not a material property but depends on the solution of humidity (and temperature). $\varepsilon_{o}^{s}(t)$ represents the deformation for $\sigma=0$, i.e., the shrinkage of an infinitesimal element unrestrained by the surrounding material. That is why $\varepsilon_{0}^{s}$ is called free shrinkage $[11,17]$. It has two components, one immediately following the humidity change and one delayed. The driving force of shrinkage comprises both the tension in free adsorbed layers and capillary water $\left(\sigma_{a}\right)$ and the decrease of the pressure in the hindered layers $\left(\sigma_{a d}\right)$. Practically, in bodies thicker than about $15 \mathrm{~cm}$, the condition $\sigma=0$ can hardly ever be met because water diffusion is so slow $[56,57]$ that a uniform distribution of humidity cannot be achieved. Thus the calculation of the actual shrinkage always represents a stress problem with creep.

For a more accurate representation of the material we may consider the model in figure $5 b$, with a series of Powers' units of different parameters $K_{c_{i}}, K_{d_{i}}, K_{f_{i}}, \varphi_{i}, \sigma_{a d_{i}}, f_{d_{i}}, i=1,2, \ldots$ In such a case, a larger system of first order differential equations would be obtained instead of (25), (26). For two Powers' units (two sorption elements) it has the form:

$$
\left.\begin{array}{l}
\dot{\varepsilon}=\left(k_{b}^{-1}+k_{c_{1}}^{-1}+K_{c_{2}}^{-1}\right)\left(\dot{\sigma}-\dot{\sigma}_{a}\right)-\dot{\sigma}_{d_{1}} / k_{c_{1}}-\sigma_{d_{2}} / k_{c_{2}}, \\
\left(\dot{\sigma}-\dot{\sigma}_{a}-\sigma_{d_{1}}\right) / k_{c_{1}}=\dot{\sigma}_{d_{1}} / k_{d_{1}}+\varphi_{d_{1}}\left(\sigma_{d_{1}}-\sigma_{a_{1}}\right)-\sigma_{d_{1}} h / k_{f_{1}} \\
\left(\dot{\sigma}-\sigma_{a}^{*}-\sigma_{d_{2}}\right) / k_{c_{2}}=\sigma_{d_{2}} / k_{d_{2}}+\varphi_{d_{2}}\left(\sigma_{d_{2}}-\sigma_{a d_{2}}\right)-\sigma_{d_{2}} \dot{h} / k_{f_{2}}
\end{array}\right\}(32 d)
$$


The response $\varepsilon(t)$ to a prescribed $\sigma(t)$ can be easily obtained by the addition of responses of individual Powers' units whose forms are the same as that given by eqs. (32a,b). Eq. (32) remains valid, but instead of $(32 a, b)$ we have (for $\left.\sigma_{d_{i}}(0)=\sigma_{a}(0)=0\right)$ :

$$
\begin{aligned}
L(t, \tau) & =\sum_{i}\left[\frac{\tilde{\varphi_{i}} / k_{c_{i}}}{1+k_{c_{i}} / k_{d i}}\right]_{\tau} e^{-\int_{\tau}^{t} \tilde{\varphi}_{i} d t^{\prime}} \\
L_{a}(t, \tau) & =L(t, \tau)+\sum_{i}\left[\frac{\phi_{i} f_{d_{i}}}{\kappa_{c_{i}} f_{a}}\right]_{\tau} e^{-\int_{\tau}^{\tau_{\tau}} \widetilde{\varphi}_{i} d t^{\prime}}
\end{aligned}
$$

The inverse values $\tilde{\varphi}_{i}^{-1}$ have the dimension of time and may be called retardation times $\tau_{i}$, as in viscoelasticity $[64,65]$. If the values $K_{c_{i}}^{-1}$ are plotted as a function of $\tau_{i}$, we may speak about " retardation spectrum ". The reason why not only one retardation time (as for model in Fig. 5a) but a whole spectrum must be considered, is that cement paste has a random microstructure, and the shapes and locations of the hindered adsorbed layers are not the same, but are random variables with a certain statistical distribution. With respect to this statistical character, the distribution of $K_{r_{i}}^{-1}$ as a function of $\tau_{i}$ is continuous and the number of Powers' units in the chain in figure $5 b$, as well as the number of equations in $(32 d)$, is infinite.

Therefore the simple model in figure $5 a$, with just one retardation time, can be considered for only a rather limited range of the delay of response of the material. Say, we determined the numerical values of parameters in (25), (26) by fitting the creep data in the range from 1 to 10 months after load application. Then we cannot expect to get a reasonable answer for the response delay of 1 hour, or 1 week, or 5 years, unless we determine new parameters for this particular range of delay. The wider the range of response delay to be represented, the longer the chain in the model in figure $5 b$ must be considered.

\subsection{Deviatoric creep}

We must realize beforehand the fact that adsorbed water layers are characterized by orientation in space. Thus, even a distorsion at constant volume will change the volume of most individual layers, i.e. contract layers of some directions and expand layers of other direction, although the total volume of adsorbed water must remain unchanged. Therefore, the old argument that water cannot explain deviatoric creep since its volume is unchanged is not true.

It is obvious that in an unsaturated porous medium the shear stresses in the fluid (i.e. in free adsorbed layers and capillary water) are so small that the total deviatoric stresses are equal to the stresses in the solid. (In a saturated medium, our assumption of small flow rate is necessary for that conclusion.)

By suitable orientation of axes, any stress deviator $s_{i j}$ in Cartesian cordinates can be expressed in terms of normal stresses only, with zero shears. Consider the deviator

$$
s_{11}=-\tau, s_{22}=\tau, s_{33}=s_{12}=s_{23}=s_{13}=0
$$

which represents the shear stress $\tau=\tau(t)$ in the planes forming the angle $\pi / 4$ with the $x_{1}-$ and $x_{2}-$ axes. For easier conception let us consider only those hindered adsorbed layers which are parallel to one of the coordinate planes. The stresses resulting from the hindered layers of these directions again have the form

$$
s_{d_{11}}=-\tau_{d}, s_{d_{22}}=\tau_{d}, s_{d_{33}}=s_{d_{12}}=s_{d_{23}}=s_{d_{13}}=0
$$

where $\tau_{d}=\tau_{d}(t)$. Let us denote by $\varepsilon_{d_{11}}, \varepsilon_{d_{22}}, \varepsilon_{d_{33}}$, the normal strains due to contractions of each of these systems of layers, and by $e_{11 d}, e_{22 d}, e_{33 d}$, their deviatoric components. According to (17) we can write

$$
\left.\begin{array}{l}
2 \dot{\varepsilon}_{d_{11}}=\dot{s}_{d_{11}} / G_{d}+\psi_{d}\left(s_{d_{11}}+P_{a} f_{d}^{\prime}\right)-s_{d 11} \dot{h} / G_{f} \\
2 \dot{\varepsilon}_{d_{22}}=\dot{s}_{d_{22}} / G_{d}+\psi_{d}\left(s_{d_{22}}+p_{a} f_{d}^{\prime}\right)-s_{d_{22}} \dot{h} / G_{f}
\end{array}\right\}
$$

where $\mathrm{G}_{d}, \mathrm{G}_{f}, \psi_{d}, f_{d}^{\prime}$ are material constants analogous to $\mathrm{K}_{d}, \mathrm{~K}_{f}, \varphi_{d}, f_{d}$, depending on humidity. In linear isotropic bodies, the strain deviator is similar to the stress deviator

$$
e_{d_{11}}=-\gamma_{d} / 2, \quad e_{d_{22}}=\gamma_{d} / 2, e_{d_{33}}=e_{d_{12}}=e_{d_{23}}=e_{d_{13}}=0
$$

where $\gamma_{d}$ represents the angle of shear. Subtracting eqs. (34) and noting that $\varepsilon_{d_{22}}-\varepsilon_{d_{11}}=e_{d_{22}}-e_{d_{11}}$ we obtain 


$$
\dot{\gamma_{d}}=\frac{\dot{\tau}_{d}}{G_{d}}+\psi_{d} \tau_{d}-\frac{h^{\circ}}{G_{f}} \tau_{d}
$$

The condition of isotropy (and Onsager reciprocity relationships (63)) requires that the same relationship be valid between all corresponding deviatoric components $e_{d_{i j}}$ and $s_{d_{i j}}$. Then, subtracting their sum from each of eqs. (34), we obtain

$$
2 \dot{e}_{d_{i j}}=\frac{\dot{s}_{d_{i j}}}{G_{d}}+\psi_{d} s_{d_{i j}}-\frac{\dot{h}}{G_{f}} s_{d_{i j}} \quad(i, j=1,2,3) \quad \text { (37) }
$$

To make clear the significance of $e_{d_{i j}}\left[\right.$ or $s_{d_{i j}}$, we must emphazise that $e_{d_{12}}\left[s_{d_{12}}\right]$ does not represent the shear strain [shear stress] in the hindered adsorbed layers perpendicular to $x_{1}$ and $x_{2}$, but the contraction or extension [pressure] in the layers which form the angle $\pi / 4$ with $x_{1}$ and $x_{2}$ (Fig. 6a). What happens to a layer forming an arbitrary angle with the axes of principle strains can be determined by a coordinate transformation of $e_{d_{i j}}$ and $s_{d_{i j}}$ (rotation) such that this layer becomes perpendicular to some principal strain or parallel to some maximum shear plane.

We can proceed further in an analogous manner as for volumetric deformation. Assuming the three-element model for deviatoric creep, which can be instructively represented as in figure 6 , we may write, in analogy with eqs. (25), (26),

$$
\begin{aligned}
& 2 \dot{e}_{i j}=\frac{\dot{s}_{i j}}{G}+\frac{\psi}{G_{c}} s_{d_{i j}}-\frac{\hbar}{G_{h}} s_{d i j}, \\
& \frac{\dot{s}_{d_{i j}}}{G_{c}}=\frac{\dot{s}_{i j}}{G_{0}}-2 \dot{e}_{i j} \quad(i, j=1,2,3)
\end{aligned}
$$

where

$$
\left.\begin{array}{l}
G_{a}=\left(G_{b}^{-1}+G_{c}^{-1}\right)^{-1}, G=\left[G_{b}^{-1}+\left(G_{c}+G_{d}\right)^{-1}\right]^{-1} \\
\psi=\left[v_{a} a_{1} /\left(f_{d}^{\prime 2} \bar{d}^{\prime}\right)\right] G_{c} /\left(1+G_{c} / G_{d}\right), \\
G_{h}=\left(1+G_{c} / G_{d}\right) v_{a} f_{d}^{-2}\left(\beta_{a} \bar{\delta}_{d} d f_{d} / d h\right)^{-1}
\end{array}\right\}
$$
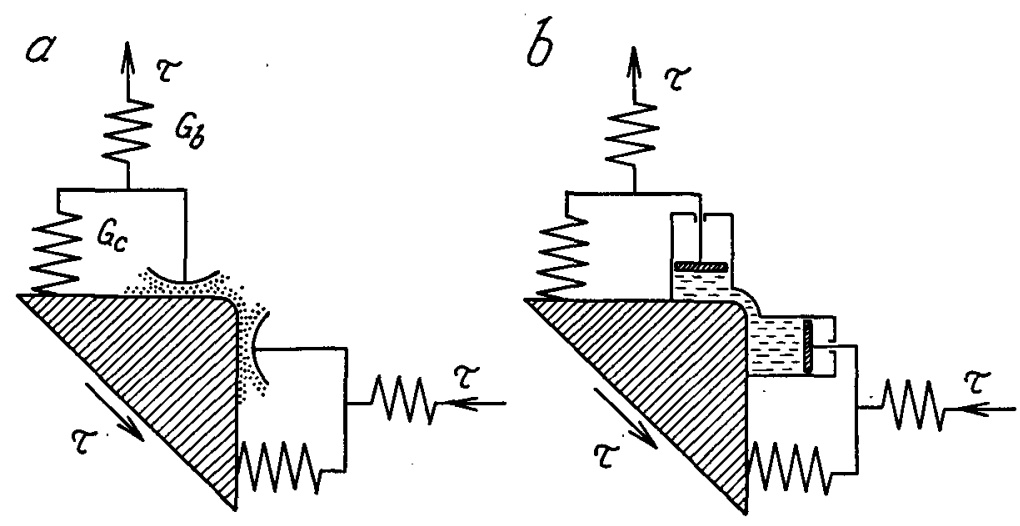

FIG. 6. - Models helping to visualize the mechanism of shear creep (corresponding to fig. $5 a$ ). $a$ with coupled sorption elements, $b$ model equivalent to $a$, with two interconnected pistons (which are equivalent to a dashpot).

It is worth noting that a formally equivalent model may be formed of pistons connected by tubes of variable size, as shown in figure $6 b$ (or of dashpots). There is no reason for the rate coefficient $\psi$ to be equal to $\varphi$.

We need not to introduce here the further procedure in which equations analogous to (30)-(32) may be obtained (and the deviatoric stress memory function defined), the only difference being that the terms $\sigma_{a}, \sigma_{a d}$ or $\sigma_{a d_{i}}$ would be missing. 


\section{VARIABLE TEMPERATUKE}

\subsection{Desorption-sorption isotherms}

To make eq. (4) for the desorption isotherm valid at different temperatures, we must write the evaporable water content $w_{e}$ instead of the total water content $w$ since the nonevaporable water content $w_{n}$ decreases with temperature. For variable temperature the full expression is

$$
d h=k(h) v_{c} d w_{e}+\left(\frac{\partial h}{\partial T}\right)_{w_{e}, t_{e}} d T+d h_{s}\left(t_{e}\right)
$$

which can be rearranged as follows

$$
\begin{gathered}
d h=k(h) v_{c} d w+x(h) d T+d h_{s}\left(t_{e}\right) \\
\text { where } \\
x(h)=\left(\frac{\partial h}{\partial T}\right)_{w_{e}, t_{e}}-k(h) v_{c}\left(\frac{\partial w_{n}}{\partial T}\right)_{t_{e}}=\left(\frac{\partial h}{\partial T}\right)_{w, t_{e}}
\end{gathered}
$$

$\varkappa$ will be called the hygrothermic coefficient. It represents the change of humidity for $1^{\circ} \mathrm{C}$ at a fixed total water content, a quantity which is directly measurable. Experimental evidence shows that it is always positive, which agrees with the fact that equilibrium water content $w$ decreases with $T$ at constant $h$ [66]. From the results of measurements on a certain mortar [67] it has been determined that $x \approx 0.0056 /{ }^{\circ} \mathrm{C}$ at $\mathrm{T}=36^{\circ} \mathrm{C}$ and $h=0.54$. Eq. (42) describes the family of desorption isotherms at different temperatures.

The form of the function $x=\varkappa(h)$ for about $h<0.4$ may be estimated according to the B.E.T. equation [43] for free adsorption in multimolecular layers (if we neglect the deviations due to hindered adsorption and capillary water). This equation is

$$
w_{e} / w_{m}=(1-h)^{-1}-(1+C, h)^{-1}
$$

where $\mathrm{C}_{1}=c_{1} e^{\left.\Delta \mathrm{Qac}_{\mathrm{a}} / \mathrm{RT}\right)}-1, c_{1} \approx 1, w_{m}=$ mass of the adsorbed layer one molecule thick, and $\Delta \mathbf{Q}_{a c}=$ differential latent heat of adsorption [42]. Differentiating eq. (43) with respect to $\mathrm{T}$ at constant $w_{e}$, we obtain, after rearrangement

$$
x=x_{0} \frac{h(1-h)^{2}}{1+C_{1} h^{2}}-k(h) v_{c}\left(\frac{\partial w_{h}}{\partial T}\right)_{t_{e}}
$$

where $x_{o}=\Delta Q_{a c} /\left(R^{2}\right)$. We see that expression (44) is indeed always positive. It even satisfies the obvious limit condition for $h \rightarrow 0$, that is, $x \rightarrow 0$. For $h=1$ and $d T>0, x=0$. (An expression better satisfying the fact $[68-70,2]$ that thermal swelling attains maximum for $h=0.7$ is in eq. (60).) $x$ may be irreversible for similar reasons as $k$.

Note. The effect of capillary water alone. At constant $w_{c}$ we may assume $r_{1}, r_{2}=$ const. in eq. (2a). With respect to eq. (1) we obtain $\ln h \sim-\gamma / \mathrm{T}$ or $d h \sim-d(\gamma / \mathrm{T}) / h$. Because $d \gamma / d \mathrm{~T}<0([44]$, p. 46$), d h>0$.

\subsection{Diffusion of water in concrete}

The rate of diffusion in the adsorbed layers grows with temperature, that is the coefficients $b_{\text {r }}$ and $a_{\mathrm{T}}$ increase. They have to obey the Arrhenius equation for thermally activated processes [71, 72]

$$
b_{T}=b e^{-Q /(R T)}, \quad a_{T}=a e^{-Q /(R T)}
$$

where $\mathrm{T}=$ absolute temperature, $a, b, \mathrm{R}$ are constants and $\mathrm{Q}, \mathrm{Q}^{\prime}$ are activation energies $[71,72]$ (enthalpies). For $h=1$, expression (45) for $b_{\mathrm{T}}$ has been verified experimentally [58]; from these results we can calculate $Q^{\prime} / R=3600^{\circ} \mathrm{K}$. (For hydrated $C_{3} S, Q^{\prime} / R=3650^{\circ} \mathrm{K}, Q / R=9720^{\circ} \mathrm{K}[73]$.)

However, recent measurements, showing that $Q^{\prime}$ depends on the average pore size [94], rather suggest that $Q^{\prime} / R=5700^{\circ} \mathrm{K}$ at $h=1,23.5^{\circ} \mathrm{C}$ and porosity 0.28 .

Furthermore, the substitution for $w$ from eq. (41) into eq. (7c) results in the following equation, instead of eq. (8),

$$
\frac{\partial h}{\partial t}-\frac{\partial h^{\prime}}{\partial t}=k(h) \frac{\partial}{\partial x}\left(\delta_{T} c(h) \frac{\partial h}{\partial x}\right)
$$

where

$$
\frac{\partial h^{\prime}}{\partial t}=\frac{\partial h_{s}\left(t_{e}\right)}{\partial t}+\partial(h) \frac{\partial T}{\partial t} \quad(460)
$$




\subsection{Deformations}

If the solution $h=h(x, t)$ of the diffusion equation (45) at a variable temperature $\mathbf{T}$ is known, the stress in the fluid, $\sigma_{a}$, and the equilibrium stress, $\sigma_{a d}$, in the load-bearing water may be determined from eqs. (1), (20), (22). The deformation caused by this change of $\sigma_{a}$ and $\sigma_{a d}$ with $\mathrm{T}$ is already included in eqs. (25), (26). This deformation will be called hygrothermic dilatation. Its immediate component is introduced by $\sigma_{a}$; its component which is delayed with respect to a change in $T$ (inside specimen) is due to $\sigma_{a d}$. Upon return to the same humidity this dilatation is partly irreversible, probably for the same reasons as is shrinkage. (See Appendix C7).

There is also another factor influencing $\sigma_{a d}$. At variable $T$, the diffusion rate in eq. (16) does not depend on $p_{d}-p_{a}$, but rather upon the difference $G_{d}-G_{a}$ in partial Gibb's free energies, as is explained in Appendix A (eq. A2). Let us assume that the material has been initially in equilibrium at $T=T_{0}$, $h=1, \sigma=0$. According to (A1) (for $\mathrm{T}$ close to $\left.\mathrm{T}_{0}\right):$ where $\mathrm{S}_{a}-\mathrm{S}_{d}=\left(\mathrm{Q}_{d}-\mathrm{Q}_{a}\right) / \mathrm{T}_{0}$ is the difference in partial entropies; $Q_{d}-Q_{a}$ is the difference in latent heat of adsorption between the hindered and unhindered adsorbed layers, due to a difference in their average thickness $\delta_{d}$ and $\delta_{a}\left(Q_{d}>Q_{a}\right)$. By the same procedure as used for the derivation of eq. (17), we could find that the following term must be added to the expression (20) for $\sigma_{a d}$ :

$$
\begin{gathered}
G_{d}^{1}-G_{a}^{1}=\left(S_{a}-S_{d}\right)\left(T-T_{0}\right)+v_{d}\left(P_{d}-P_{a}\right) \\
\left(1-T / T_{0}\right)\left(Q_{d}-Q_{a}\right) v_{a} / f_{d}
\end{gathered}
$$

The dilatation due to this term will be called thermal swelling (or thermal shrinkage). It causes a delayed partial recovery of deformation after a change in $\mathrm{T}$ and has no immediate component.

The third effect to be included is the change of specific volumes of microscopic constituents at constant pressure, i.e. pure thermal dilatation. Thus to the right-hand sides of eqs. (23), (24), (17) the following terms must be added

$$
\alpha_{b} \dot{T}, \alpha_{c} \dot{T}, \quad \alpha_{d} \dot{T}
$$

where $\alpha_{d}$ is the thermal expansion coefficient for the thickness $\bar{\delta}_{d}$ of the adsorbed water layers at a constant mass per unit surface and constant $p_{a} \cdot \quad \alpha_{b}, \alpha_{c}$ are the coefficients for the two springs in figure $5 a$. It may be shown, in the same way as eqs. (25), (26) were deduced, that

$$
\begin{aligned}
\dot{\varepsilon}-\alpha_{1} \dot{T} & =\frac{\dot{\sigma}-\dot{\sigma}_{a}}{K}+\frac{\varphi}{K_{c}}\left(\sigma_{d}-\sigma_{a d}\right)-\frac{\dot{h}-x \dot{T}}{K_{h}} \sigma_{d} \\
\frac{\dot{\sigma}_{d}}{K_{c}} & =\frac{\dot{\sigma}-\dot{\sigma}_{a}}{K_{a}}-\dot{\varepsilon}+\alpha_{o} \dot{T}
\end{aligned}
$$

where $\alpha_{o}=\alpha_{b}+\alpha_{c}, \alpha_{1}=\alpha_{o}+\left(\alpha_{d}-\alpha_{c}\right) /\left(1+K_{c} / K_{d}\right)$. The last term in eq. (48) needs explanation. In eq. (25) for constant $\mathrm{T}, \dot{h}$ appeared because we put $\dot{f}_{d}=\dot{h}\left(d f_{d} / d h\right)$. In case of variable $\mathrm{T}$, however, $h$ varies even if $w$ is kept constant which means that $f_{d}$ is constant too. With respect to (42), we must thus replace $\dot{h}$ by $\dot{h}_{e q}-\varkappa \dot{\mathrm{T}}$. $h_{e q}$ represents the equivalent humidity (at reference temperature $\mathrm{T}_{0}$ ) which gives the same water content $w$ (at the same $t_{e}$ ).

Note. At variable $\mathbf{T}$ the parameters $\varphi, \psi, \varphi_{i}, \mathrm{~K}_{h}, \mathrm{~K}_{f_{i}}, c, \mathrm{C}, f_{d}, f_{d_{i}}$ must be considered as functions of $w$, rather than $h$. At variable $t_{e}$ and $\mathrm{T}$, they should be considered as functions of $h_{e q}$ (while $\beta$ and $p_{a}$ remain functions of $h$ ).

Note. Approximating, we can put $\alpha_{c}=\bar{\delta}_{d} \alpha_{c e m}, \alpha_{b}=\left(1-\bar{\delta}_{d}\right) \alpha_{c e m}$ and $\alpha_{d}=\bar{\delta}_{d} \alpha_{w}$, where $\alpha_{c e m}=\alpha_{o}$ is the coefficient of linear expansion of dried cement paste (which is probably less than $11 \times 10^{-6}$ per ${ }^{0} \mathrm{C}$ ) and $\alpha_{w}$ is that for the adsorbed water (for liquid water between $10^{\circ} \mathrm{C}$ and $30^{\circ} \mathrm{C}$ about $66 \times 10^{-6}$ per ${ }^{\circ} \mathrm{C}$ ). Since $\alpha_{w}>\alpha_{c e m}, \alpha_{1}$ must increase with the area factor, i.e., with humidity. Perhaps $\alpha_{1} \approx \alpha_{o}+\left(\alpha_{1}-\alpha_{o}\right) h$.

In eq. (31) the following term has to be added to the right-hand side (assuming $\mathrm{T}=\mathrm{T}_{o}$ for $t=0$ ):

$$
\left(K_{c} / \tilde{\varphi}\right) \alpha, \dot{T}+K_{c} \alpha_{0}\left(T-T_{0}\right)
$$

In eq. (32) we would have to add at the right hand side the term

$$
\alpha, \Delta T(t)+\int_{0}^{t} \Delta T(\tau) L_{T}(t, \tau) d \tau
$$


where

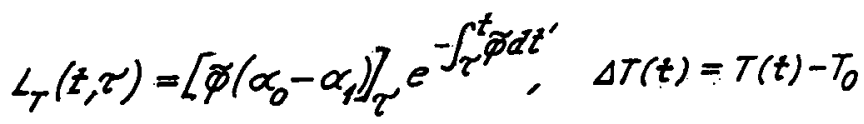

$\mathbf{L}_{\mathbf{T}}$ may be called the temperature memory function; $\tilde{\varphi}=\varphi-(\dot{h}-x \dot{\mathrm{T}}) \mathrm{K}_{c} / \mathbf{K}_{h}$.

Another effect, and the only one which affects creep directly, is the variation of the rate constants $\varphi$ and $\psi$ with $\mathrm{T}[18,74,12,91,92,103]$. In eqs. (19) and (40), this effect is given by $a_{\mathrm{T}}$ according to eq. (45). At constant $w$ the changes of $f_{d}, \bar{d}, f_{d}^{\prime}, \bar{d}^{\prime}$ will probably be negligible, so that $\varphi \sim a_{\mathrm{T}} \sim \psi$. According to some tests $[18,74]$, it may be estimated that $Q / R \approx 2800^{\circ} \mathrm{K}$.

Note. The decrease of elastic moduli with temperature $[75,12]$ is probably only apparent and is caused by accelerated creep (as in polymers [64]).

\subsection{Aging}

Acceleration of hydration, caused by increase of temperature [76-78], is introduced by the coefficient $\beta_{T}$ in eq. (4) for the equivalent curing period. Since the rate of hydration as a chemical reaction is governed by the Arrhenius equation [71, 72, 79], we have

$$
\beta_{T}=\beta(h) e^{-q /(R T)}
$$

where $\beta$ is a function of $h$ only. If only one process is involved, the activation energy $q$ is constant; when more processes are effective, the apparent $q$ might be variable with $T$. Nevertheless, constancy of $q$ was found by experiments [78] (between $4^{\circ} \mathrm{C}$ and $110^{\circ} \mathrm{C}$ ); it might be calculated from these experiments that $q / \mathrm{R}=2500^{\circ} \mathrm{K}$.

\section{IRREVERSIBLE CREEP AND SHRINKAGE}

To some extent the irreversibility of creep and shrinkage is caused by the increase of $K_{c}$ and $G_{c}$ with $t_{e}$ as the concrete is aging. This irreversibility is included in the linear creep law (25), (26), etc. It disappears, however, after the hydration stops whereas a large part of creep is still known to be irreversible. Assuming that the limiting value of creep for $t \rightarrow \infty$ under constant load is bounded, we must conclude that there must also exist some other source of irreversibility which must be nonlinear, i.e. violating the principle of superposition in time.

Comparing the structure of cement gel with that of metals, there seems to be little reason for any plasticity. This observation is reinforced by the fact that irreversible creep appears even for very small stresses at which the creep depends linearly on stress. Even the curvature and irreversibility at low stress of the stress-strain diagram for short-time tests may be attributed to a short-time linear creep [12, 100] (the same as in polymers [64]), rather than to plasticity.

For the above reasons, it has been suggested $[80,23,22]$ to allow for the irreversibility by a rheological model shown in figure $5 e$. This model contains a new rheological element-the ratchet (with a pawl)-instead of the Saint Venant element for plasticity. During an increase in deformation, the additional spring is out of action. Upon reversal of deformation (recovery), the ratchet snaps down, rigidly connecting the additional spring. The ratchet may represent the formation of new chemical bonds between the solid surfaces (the possibility of which was proved by compacting isolated hydrated cement particles into a solid body [81]), the partly irreversible dissolution of solid matter under load (Appendix C10), or the irreversible decomposition of the interlayer hydrate [81] under load (Appendix C8).

The mathematical formulation is simpler than in the case of plasticity. Eqs. (25)., (26) remain valid as long as the condition for the increase of creep deformation is not violated. For volumetric creep this condition is

$$
d \sigma_{c}^{2} / d t \geq 0 \text { where } \sigma_{c}=\sigma-\sigma_{d}-\sigma_{a}
$$

For deviatoric creep this condition must comply with the requirements of isotropy, i.e. it may involve only the second (or third) invariant of $s_{i j}-s_{d_{i j}}$. Then the condition is

$$
\dot{I}_{2} \geq 0 \text { where } I_{2}=\frac{1}{2} \sum_{i} \sum_{j}\left(s_{i j}-s_{d_{i j}}\right)\left(s_{j i}-s_{d_{j i}}\right)
$$

When some of the inequalities (53) become violated, which is caused by unloading (or non-proportional loading) or by change of humidity (or temperature), eqs. (25), (26) etc. will still have the same form, but greater values of $K^{\prime}{ }_{c}, G^{\prime}{ }_{c}$, and eventually also different $\varphi^{\prime}, \psi^{\prime}$, have to be substituted. It is 
an advantage that this system of equations is still linear, although the time responses to various loadings may not be superposed if the intervals of validity or invalidity of the conditions $(53 a),(53 b)$ do not coincide. It is unclear whether the condition $(53 a)$, could be used if $\sigma$ changes sign.

The third cause of irreversibility, occurring with variable humidity only, is the irreversibility of the area factors $f_{d}, f_{a}$ (and inherent irreversibility of $\sigma_{a}, \sigma_{a d}$ in case of shrinkage). This is due to the irreversibility of desorption isotherms.

An apparent irreversibility in tests of creep recovery is also obtained if the internal humidity of the samples has changed.

Note. The cyclic creep $[1,2,82,14]$, i.e. creep under stresses repeated many times, is not adequately interpreted by the ratchet and should rather be regarded as an accelerated creep, also caused by some type of nonlinearity, e.g. accumulation of second-order deformation in sorption element. For first reloading and few further cycles more complex models with ratchet may be set up.

\section{ANALYSIS OF STRESS AND STRAIN PROBLEMS}

\subsection{General method of numerical analysis}

In most practical problems the only feasible method of solution is the numerical one. The stepby-step integration in time can be formulated as a succession of initial strain problems [83]. Here we outline its principle. For each time interval $\Delta t$, eqs. (48), (49) and (38), (39) may be replaced by the difference equations

$$
\begin{aligned}
& \Delta \varepsilon=\frac{\Delta \sigma}{K}+\varepsilon^{0} \\
& \Delta e_{i j}=\frac{\Delta s_{i j}}{2 G}+e_{i j}^{0}
\end{aligned}
$$

where

$$
\begin{aligned}
& \varepsilon^{0}=\frac{\phi}{K_{c}}\left(\sigma_{d}-\sigma_{a d}\right) \Delta t-\frac{\Delta \sigma_{a}}{K}+\alpha, \Delta T-\frac{\Delta h}{K_{h}} \sigma_{d} \\
& e_{i j}^{0}=\frac{\psi}{2 G_{c}} s_{d_{i j}} \Delta t-\frac{\Delta h}{G_{h}} s_{d_{i j}} \\
& \Delta \sigma_{d}=K_{c}\left(\frac{\Delta \sigma-\Delta \sigma_{a}}{K_{a}}-\Delta \varepsilon+\alpha_{0} \Delta T\right) \\
& \Delta s_{d_{i j}}=G_{c}\left(\frac{\Delta s_{i j}}{G_{a}}-2 \Delta e_{i j}\right)
\end{aligned}
$$

Assume that the solution of humidity is determined and that $\sigma, \varepsilon, \sigma_{d}, s_{i j}, e_{i j}, s_{d_{i j}}$ at the start $t_{(n)}$ of interval $\Delta t_{(n)}$ are known. Then we can compute $\varepsilon_{(n)}^{0}$ and $e_{i j(n)}^{0}$ at each point of the body. Thus eqs. (54), (56) have the form of an elastic stress-strain law with prescribed fictitious initial strains $\varepsilon_{(n)}^{0}$, $e_{i j_{(n)}^{0}}^{0}$. The solution of $\Delta \varepsilon_{(n)}, \Delta \sigma_{(n)}, \Delta e_{i j(n)}, \Delta s_{i j(n)}$ satisfying the differential equilibrium conditions for $\Delta \sigma_{(n)}$ and $\Delta s_{i j(n)}$, the geometric equations and the boundary conditions, is a known problem of elasticity. After its solution we can calculate $\Delta \sigma_{d(n)}, \Delta s_{d_{i j(n)}}$, from eqs. (56) and (59) and determine $\sigma_{(n+1)}=\sigma_{(n)}+\Delta \sigma_{(n)}$ etc. The algorithm of solution is elucidated in more detail in Appendix $D$. In geometrically complex bodies the finite element technique must be used.

\subsection{Creep and shrinkage tests and computer results for a hypothetical material}

With the theory outlined even the analysis of prisms and cylinders under simple loading is not easy. This is largely due to the unfortunate fact that the aggregate size does not allow samples sufficiently thin (less than $1 \mathrm{~mm}$ ) as to avoid variable $h$ throughout the unsealed samples. Nevertheless, if test results are to be interpreted correctly, we eannot avoid dealing with the complexity of our analysis.

A standard computer programm (Appendix D) was set up, based on our theory. It was assumed that the specimens are so long that plane cross sections remain plane and free of lateral restraint. When square prisms are considered, the ends and two opposite sides are assumed to be sealed in order to obtain unidimensional diffusion, as in an infinite slab. 
Tentatively, a hypothetical material has been found for which is achieved at least qualitative agreement with what is known today from tests of concrete. This hypothetical material is defined by the following expressions

$$
\begin{aligned}
& k_{b}=\frac{23+t_{e}}{30+t_{e}} 4 \times 10^{5}, k_{c}=\frac{10+t_{e}}{120+t_{b}} 4 \times 10^{5}, k_{c}^{\prime}=2 k_{c}, \\
& K_{d}=(1+h) 4 \times 10^{\sigma}, \quad K_{h} \rightarrow \infty, G_{h} \rightarrow \infty, \\
& G_{b}=0.23 K_{b}, G_{c}=0.442 K_{c}, G_{d}=0.5 K_{d}, G_{c}^{\prime}=2 G_{c} \text {, } \\
& \varphi=\frac{a_{T} h^{2}}{120\left(0.002+h^{3}\right)} \frac{K_{c}}{K} \text { where } h+h-x(T-25) \text { for } T \neq 25^{\circ} C \text {, } \\
& \psi=2 \varphi \frac{k}{K_{C}} \frac{G_{C}}{G}, \quad a_{T}=-3.7+635 /(160-T), \\
& d \sigma_{a}=d \sigma_{a d}=\left\{\begin{array}{l}
1360 \times 0.06 h d h \ldots \text { for } d h \leq 0, \\
1360 \times 0.04 h d h \ldots \text { for } d h>0,
\end{array}\right. \\
& h_{0}=\frac{0.93 t_{e}+15}{t_{e}+15}, h_{s}=\frac{0.85 t_{e}+15}{t_{e}+15}, \\
& \beta=\left[1+(7.5-7.5 h)^{4}\right]^{-1} a_{T} \text {, } \\
& x=\frac{0.02 h(1.02-h)}{1.25-h}, b_{T}=a_{T}, B \rightarrow \infty, \\
& \alpha_{0}=8 \times 10^{-\sigma}, \alpha_{1}=\alpha_{0}+4 \times 10^{-\sigma} h \text {, } \\
& c=0.1+h, k=1.5, k^{\prime}=2 k
\end{aligned}
$$

$\mathrm{K}_{b}, \mathrm{~K}_{c}, \mathrm{G}_{b}, \sigma_{a}, \ldots$ are given in $\mathrm{kp} / \mathrm{cm}^{2}, t, t_{e}$ in days, $\mathrm{T}$ in ${ }^{0} \mathrm{C}, \varphi, \psi$ in day ${ }^{-1}$. The results of the computer analyses are shown in figures 8-26.

Note. These results were obtained prior to the proper assessment of the influence of the term containing $h$ in eq. (25), and that is why we put in (60) $K_{h} \rightarrow \infty, G_{h} \rightarrow \infty$. With this assumption the opposite behavior between the drying and steady humidity conditions could not be reflected, and the creep increase at drying in figure 9 was obtained only because $\varphi$ in eq. (60) was assumed to be decreasing with $h$ at high $h$, according to eq. B3 or B4.

It is necessary to emphasize that, in general, the agreement with a particular experiment is not quantitative. But the large number of parameters permits a wide possibility of adjustment. It can be expected that if sufficient data are available from precisely controlled tests on one type of concrete, the parameters in eq. (60) could be selected so as to achieve a quantitative agreement with these test results.

Note. In this connection it is worthwhile to point out how useful it would be if a general agreement could be achieved about one specific type of concrete which is to be used in the experimental studies of rheological properties in all laboratories. Plotting the results of different investigators against our computed curves would bring in a tremendous scattering which is not a material property but is due to different types of concrete used, different test procedures and environmental conditions, the control of which had been inadequate in older experiments.

\section{CONCLUSION}

The results of the numerical analysis of various tests of shrinkage, creep and thermal expansion show that our theory is able to reflect correctly the known features of shrinkage, creep and thermal expansion of concrete under sustained load. 


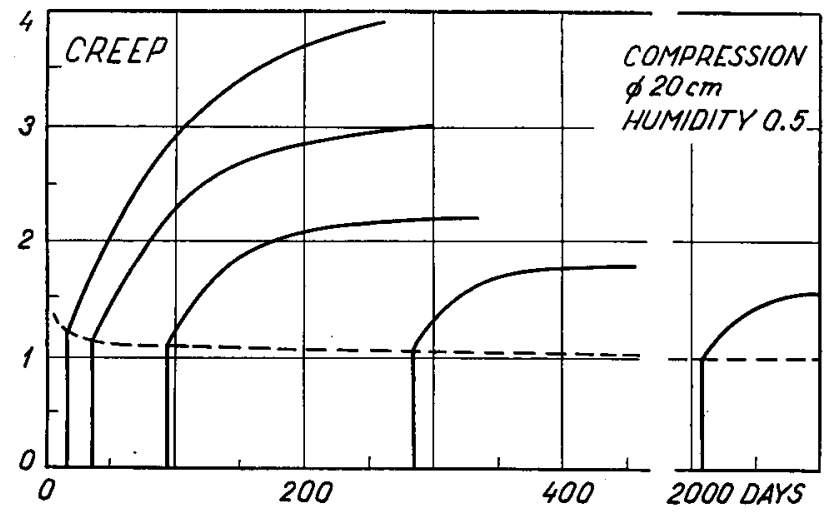

Fig. 8
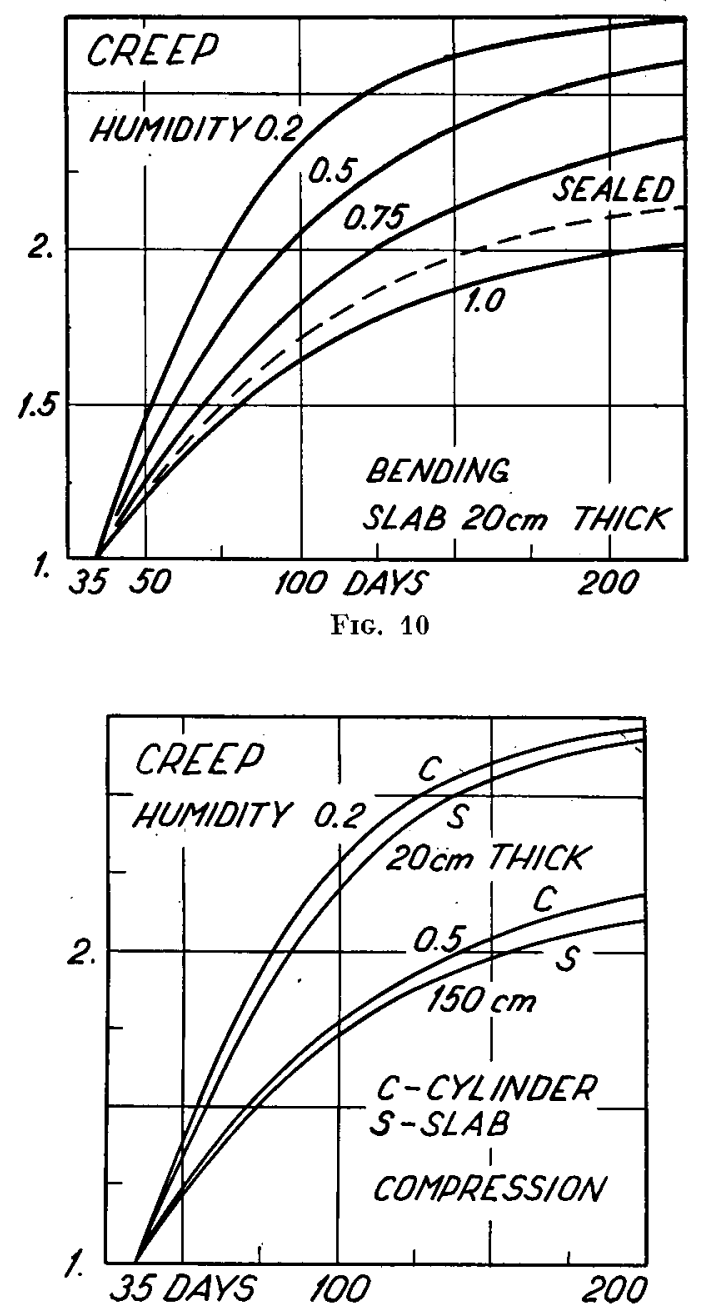

Fig. 12
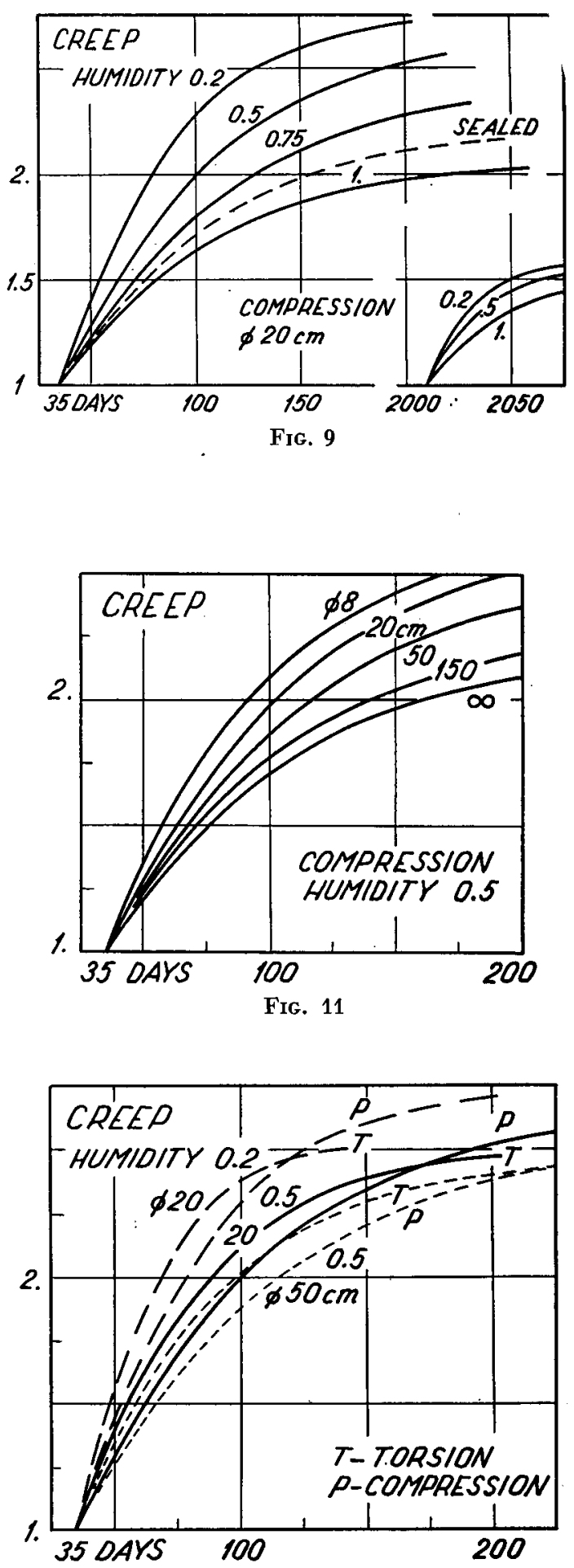

FIG. 13

Fig. 8-22. - Results of analyses of creep and shrinkage tests. Humidities indicated on figures represent ambient, not internal humidities. Creep (and relaxation) are expressed by relative values with respect to the value at sudden load application, and include the instantaneous deformation. Until the first loading, or the first change of humidity at shrinkage, all specimens are considered as being stored under water. Time scale expresses the age of concrete. $\varnothing$ indicates diameter of cylinder; slab $S$ means the prism sealed at the two sides and at the ends. On figure 16 the sample was predried (until equilibrium) after 30 days of moist curing. On figure 22, $T$ was raised immediately at the age of 35 days. 

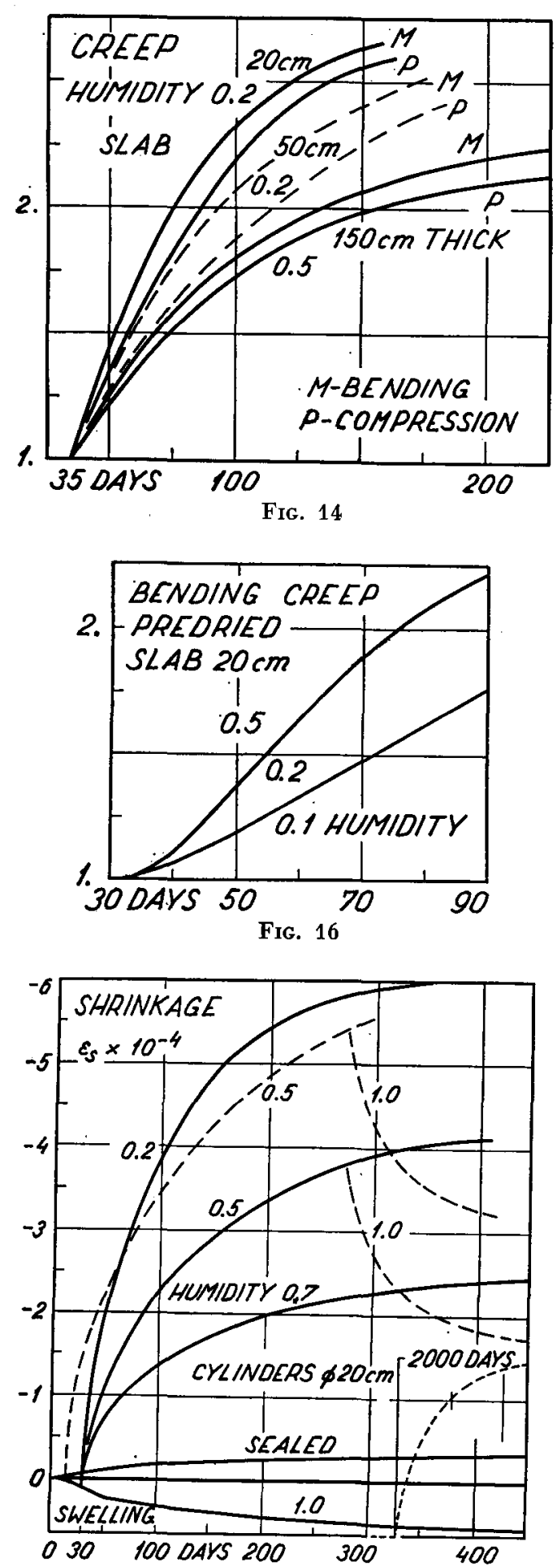

Fig. 18

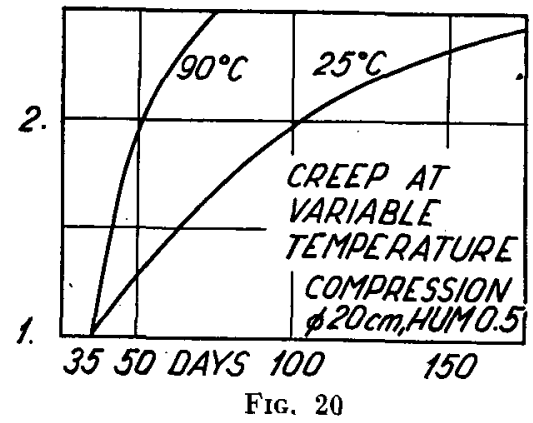

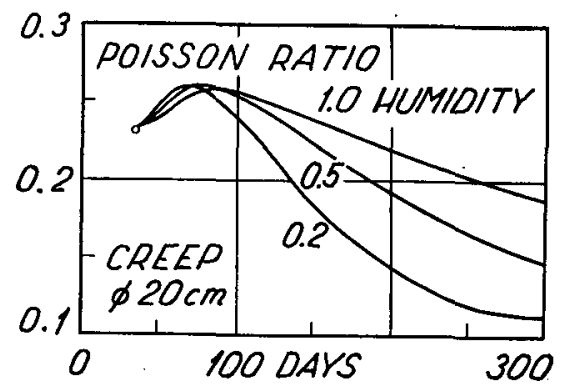

Fig. 15

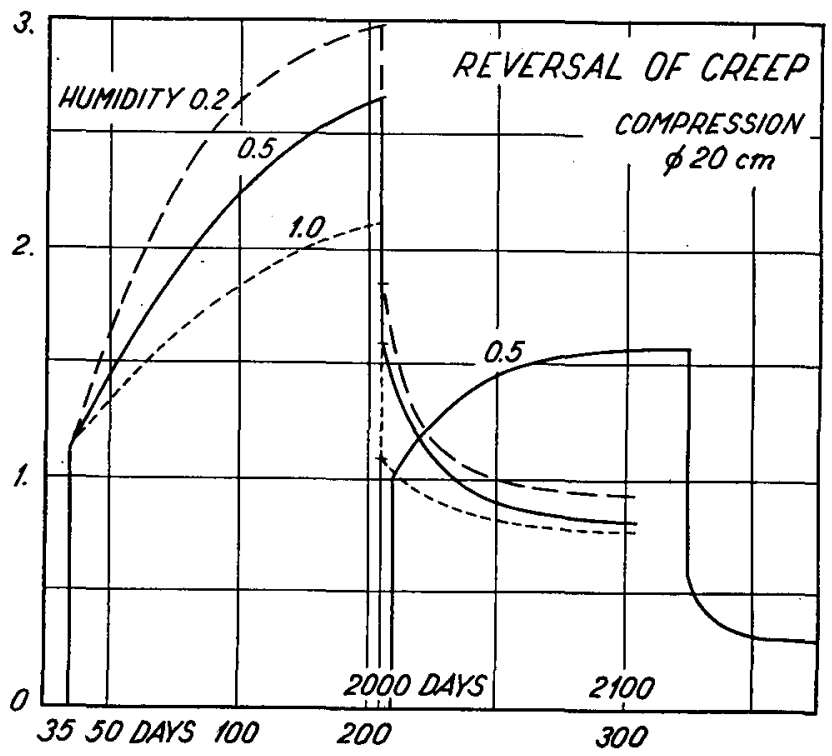

FIG. 17

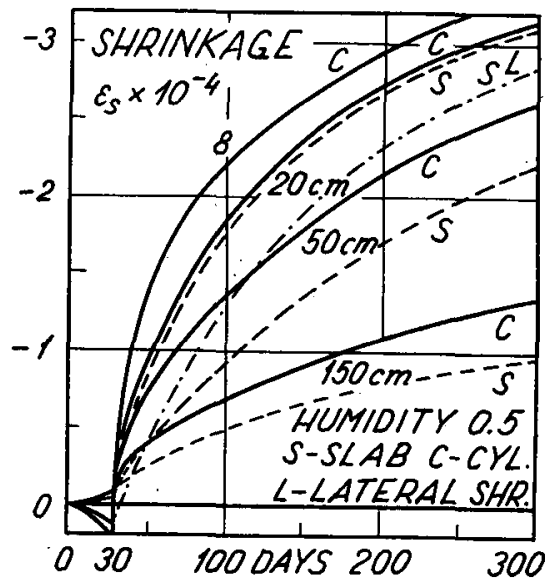

FIG. 19

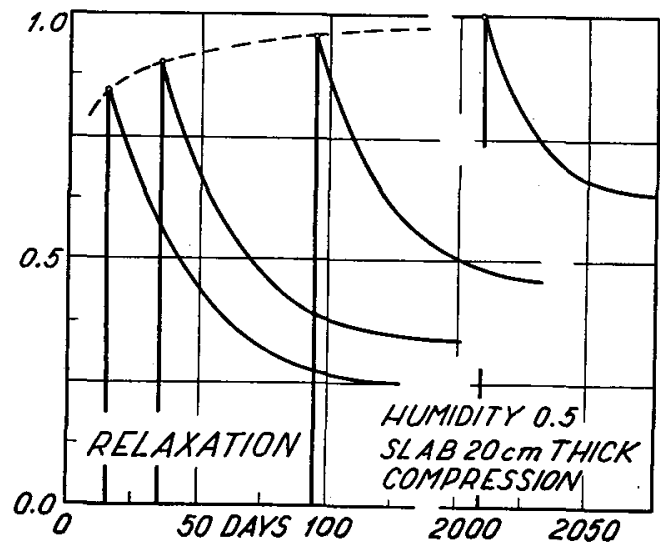

FIg. 21 

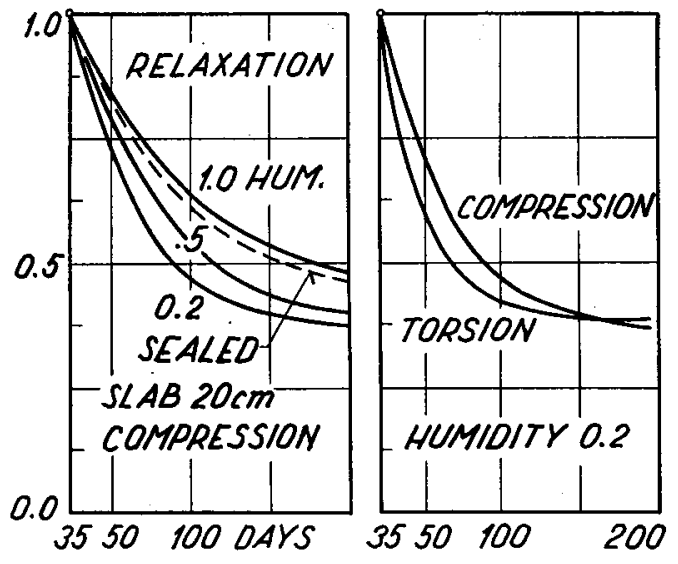

FIG. 22

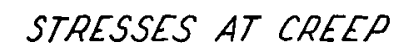

35-90 DAYS

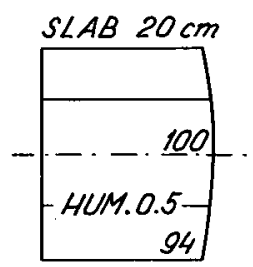

$S \angle A B 20 \mathrm{~cm}$
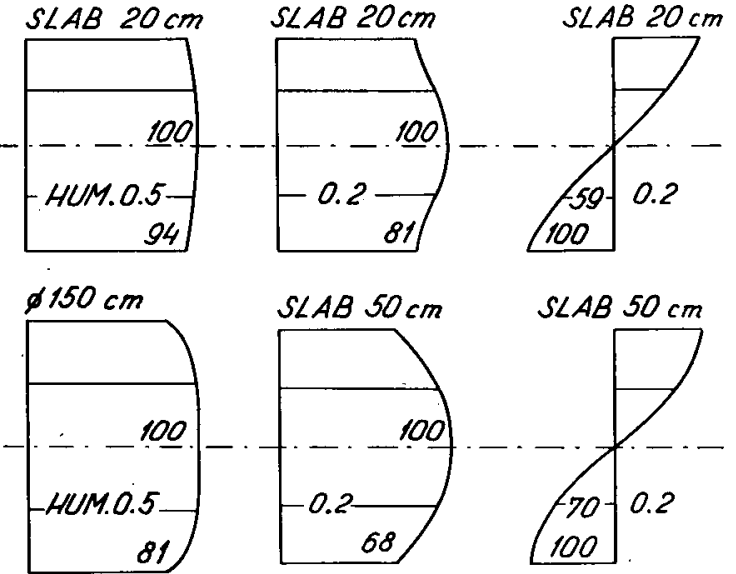

Fig. 23

Fig. 23-25. - Examples of the calculated distributions of normal stresses and humidities throughout the thickness. At creep for the age of 90 days, load applied at 35 days, at shrinkage at 45 days, ambient humidity reduced to 0.5 after 30 days of moist euring. Other conditions same as on fig. 8-22. After comparison with tests [56] we realized that the diffusion coefficient should have been considered smaller. Note the difference of shrinkage stresses in cylinders and slabs.
Fig. 8-22. - Results of analyses of creep and shrinkage tests. Humidities indicated on figures represent ambient, not internal humidities. Creep (and relaxation) are expressed by relative values with respect to the value at sudden load application, and include the instantaneous deformation. Until the first loading, or the first change of humidity at shrinkage, all specimens are considered as being stored under water. Time scale expresses the age of concrete. $\varnothing$ indicates diameter of cylinder; slab $\mathbf{S}$ means the prism sealed at the two sides and at the ends. On figure 16 the sample was predried (until equilibrium) after 30 days of moist curing. On figure 22 , $T$ was raised immediately at the age of 35 days.

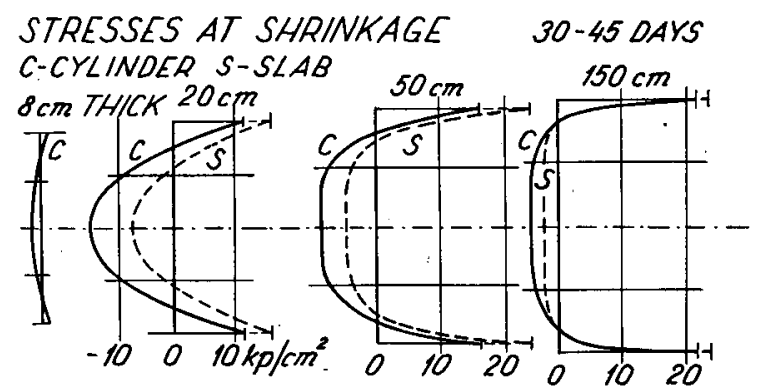

FIG. 24

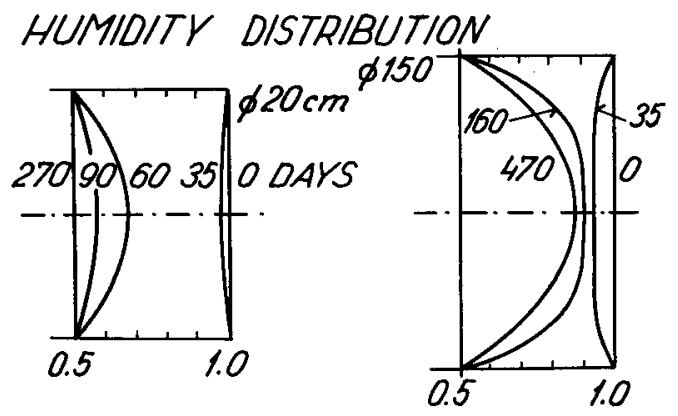

Fig. 25

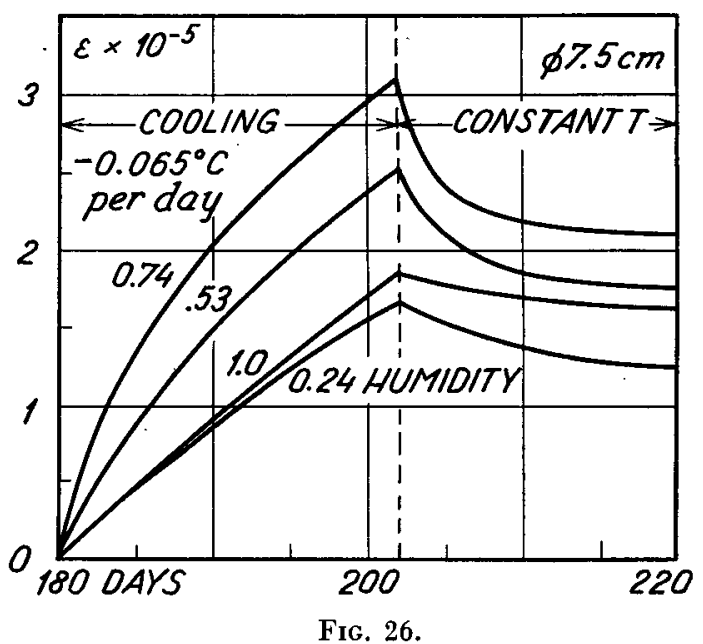

G. 20. - Computed thermal dilatation at constant rate cooling and its reversal after stoppage of cooling. ( $T$ considered approximately as constant throughout the thickness.) 


\section{APPENDIX A}

\section{Thermodynamic equilibrium of adsorbed water}

Let us choose as independent variables the intensive quantities, i.e. pressure $p$ and temperature T. Then the thermodynamic potential is the Gibbs' free energy G. For a single substance at a fixed mass content, $G$ is expressed as follows $[44,71,84]$

$$
\mathrm{G}=\mathrm{U}+p \mathrm{~V}-\mathrm{TS}
$$

where $\mathrm{V}=$ volume, $\mathrm{S}=$ entropy, $\mathrm{U}=$ total energy. This assertion may be verified if the expression for $G$ is differentiated and the relationships $d U=\delta Q-p d V, \delta Q=T d S$, expressing the first and second laws of thermodynamics, substituted. This, indeed, yields a total differential in $p$ and $\mathrm{T}$, i.e.

$$
d \mathrm{G}=\mathrm{Vdp}-\mathrm{SdT}
$$

From the condition $\delta Q>T d s$ for an irreversible process it follows that $d G \geqslant 0$, i.e. $G$ is minimized at equilibrium. Hence $d \mathrm{G}=0$ for a small deviation from equilibrium.

Note. It is seen that for $d \mathrm{~T}=0, \mathrm{G}$ coincides with the definition of complementary strain energy. The strain energy for $d T=0$ is represented by the Helmotz free energy $\mathrm{F}=\mathrm{U}$ - TS. For $\delta \mathrm{Q}=d \mathrm{~S}=0$ the place of $\mathrm{G}$ (or $\mathrm{F})$ is taken by the enthalpy $\mathrm{H}=\mathrm{U}+$ $p \mathrm{~V}$ (or by $\mathrm{U}$ ).

Consider now a single substance-water in two phases as adsorbed water and vapor which are in equilibrium. Since $G$ is independent of extensive properties such as $V$, we may write $G=$ $w_{v} G_{v}^{1}+w_{a} G_{a}^{1}$ where $G_{a}^{1}$ and $G_{v}^{1}$ denote partial free energies (per unit of quantity of each phase which may be taken as a unit mass) and $w_{a}, w_{v}$ represent the masses of the adsorbed water and vapor, respectively. Let us consider a very small deviation from equilibrium, at which the small amount $d w_{d}=-d w_{v}$ is transferred from the vapor to the adsorbed state. Since $d G=G_{v}^{1} d w_{v}+G_{a}^{1} d w_{a}=0$, we have the following equilibrium condition :

$$
\mathrm{G}_{a}^{1}=\mathrm{G}_{v}^{\mathbf{1}}
$$

This equation may be used to determine the pressure $p_{a}$ in the adsorbed film, which is in equilibrium with the ambient vapor. For this purpose we assume for water vapor the ideal gas equation

$$
p_{v} v_{v}=\mathrm{RT} / \mathrm{M}
$$

where $v_{v}=$ specific volume of vapor. For an equilibrium change at a constant temperature $T$ it follows from eq. (A1) (with $d G_{v}^{1}=v_{v} d p_{v}$ ) and eq. (A2) that

$$
d \mathrm{G}_{a}^{1}=v_{a} d p_{a}=\frac{\mathrm{RT}}{\mathrm{M}} d\left(\ln p_{v}\right)
$$

Making the assumption $v_{a} \approx$ const. (which is certainly inadmissible for $h<0.12$ ), the integration of eq. (A4) with the initial condition $p_{a}=0$ at $p_{v}=p_{\text {sat }}$ yields eq. (1).

Note. A completely analogous procedure yields for $p_{c}$ an expression of form (1) in which $v_{c}$ replaces $v_{a}$. This expression is exact because $v_{c}$, unlike $v_{a}$, may be taken as exactly constant. The equality $p_{a}=p_{c}$ is exact only at the contact with the adsorbed water.

Now let us consider a hindered adsorbed layer and imagine an equilibrium process at constant $T$ in which the ambient humidity $h$ is gradually increased while the gap thickness is kept constant. The differential equation (A4) is still valid for $p_{d}$ (in place of $p_{a}$ ) but for the initial condition we may consider the state in which the gap available becomes just full of water, i.e. $w_{a}=w_{d}$. In this state obviously $p_{d}=p_{a}$. Noting that the changes of partial Gibbs'free energies are equal, we have the differential equation $v_{a} d p_{a}=v_{d} d p_{d}$ where $v_{a}$ and $v_{d}$ are same functions of $p_{a}$ and $p_{d}$, that is $v_{a}=f\left(p_{a}\right)$, $v_{d}=f\left(p_{d}\right)$. By integration, eq. (2) is found to be valid even for $w_{a} \geqslant w_{d}$. Because $p_{d}=p_{a}$ and $\mathbf{P}_{d}=0$ at $w_{a}=w_{d}$, and noting that $d \mathbf{P}_{d}=d p_{d}$, the disjoining pressure is

$$
\mathrm{P}_{d}=p_{d}-\left[p_{a}\right]_{w_{d}}=\frac{\mathrm{RT}}{\mathrm{M} v_{a}} \int_{w_{d}}^{w_{a}} \frac{\partial(\ln h)}{\partial w_{a}^{\prime}} d w_{a}^{\prime} \quad\left(w_{a}>w_{d}\right)
$$

where $w_{a}$ is the value for the adjacent unhindered layer in equilibrium.

Instead of considering the change of vapor pressure $p_{v}$ at a constant water content $w_{a}=w_{d}$, we could have imagined a gradual decrease of $w_{a}$ at constant $p_{v}$ and T. For this purpose eq. (Ä1) first has to be generalized. In the case of variable mass content we can write

$$
\mathrm{G}_{a}=w_{a} \mu_{a p a}{ }^{\mathrm{T}}
$$

where $\mu_{a}$ is called chemical potential. The total differential of eq. (A6) is

$$
d G_{a}=w_{a} d \mu_{a}+\mu_{a} d w_{a}
$$


Because $\mu_{a}$ is independent of $w_{a}$, it represents the partial Gibbs' free energy per unit mass, i.e.

$$
\mu_{a}=\left(\partial G_{a} / \partial w_{a}\right)_{p a^{\prime}}
$$

Then $w_{a} d \mu_{a}$ must be equal to the expression (A1),

$$
w_{a} d \mu_{a}=\mathrm{V}_{a} d p_{a}-\mathrm{S}_{a} d \mathrm{~T}
$$

Consequently, we get

$$
d \mathrm{G}_{a}=\mathrm{V}_{a} d p_{a}-\mathrm{S}_{a} d \mathrm{~T}+\mu_{a} d w_{a}
$$

According to eq. (A4) where $G_{a}^{1}=\partial G_{a} / \partial w_{a}$, we have per unit of mass:

$$
\mu_{a}=\frac{\mathbf{R T}}{\mathbf{M}} \frac{\partial\left(\ln p_{v}\right)}{\partial w_{a}}
$$

Now eq. (A9) has to be rewritten for the mass (per unit solid surface) of the hindered adsorbed layer, replacing $p_{a}$ by $p_{d}$ etc. Then we consider the gradual decrease of the water amount $w_{a}$ and integrate over $p_{d}$ with the conditions $d \mathrm{G}_{d}^{1}=0, d \mathrm{~T}=0$. As a result eq. (A5) is obtained.

Note. Eqs. (1), (2), (A5) give only a simplified, though easily understandable picture. The constancy of $v_{a}($ for $h \gg 0)$ is merely an assumption. The actual dependence of $v_{\alpha}$ upon $h$ is not known. "Nevertheless, it would have been possible to arrive at the same constitutive equation with any form of the monotonic function $p_{a}=p_{a}(h)$, or even without introducing $p_{a}$ and $p_{d}$ at all, using directly the partial free energies $G_{a}^{1}$ and $G_{d}^{1}$ in eqs. (25), (26), (20). ( $\mathrm{G}_{d}^{1}-\mathrm{G}_{a}^{1}$ is the primary driving force of diffusion in eq. 16 , rather than $p_{d}-p_{a}$.) Such an approach would also eliminate another deficiency : the stress tensor in adsorbed layers has been tacitly assumed as isotropic which is certainly contrary to fact; $p_{d}$ should also be understood as the average pressure for a great number of layers $\left(p_{d}=\sigma_{d} / f_{d}\right)$ because the method of thermodynamic potentials predicts only the average behavior of sufficiently large ensembles of molecules. The application of statistical quantum mechanics would be more fundamental, especially for calculations of $\varphi=\varphi(h)$ in Appendix B.

Note. The existence of load-bearing water and disjoining pressure has been denied by some researchers. This would hold true if water completely filling thin pores behaved like a solid body with a permanent lattice. Then the equilibrium condition $d G=0$ could not be reduced to $G_{a}^{1}=G_{d}^{1}$ (or equivalent eq. 2). This relationship results by considering (as for (A2)) a small deviation from equilibrium at which the amount $d w_{d}=$ $\longrightarrow d w_{a}$ is transferred from $w_{d}$ to $w_{a}$. Thus the above denial means denying the ability of molecules to diffuse along the hindered layers. No doubt this ability exists since water in hindered layers can evaporate (and also because molecules can migrate along the adsorbed layers even without leaving them [41]). Moreover, it is inconceivable that the difference $G_{d}^{1}-G_{a}^{1}$ due to a change in $G_{a}^{1}$, as in the case of drying, would not be equivalent to that due to a change in $G_{d}^{1}$, as in the case of a change in $p_{d}$. Therefore, the above objection is unfounded.

\section{APPENDIX B}

\section{Flow in hindered adsorbed layers of various shapes}

Let us now assume for simplicity that the hindered adsorbed water flows like an incompressible fluid and that $h$ and $f_{d}$ are constant.

B1. - The layer between two solid spheres almost in contact. The variation of the layer thickness is $\delta_{d} \sim x^{2}$ where $x=$ distance from the contact point and the sign $\sim$ denotes proportionality. Let the solid spheres approach one another at the speed (Fig. 1) $\dot{\delta}_{o}=d \delta_{d} / d t$. Then $v \delta_{d} \sim x$ where $v$ is the radial velocity of the flow of the adsorbed molecules. The pressure gradient in the layer is $\partial p_{d} / \partial x \sim\left(v \delta_{d}\right) / \delta_{d}^{n+1} \sim x^{-2 n-1}$ where $n$ is the constant in eq. (10). By integration from $x=0$ to $x=x_{a}$, where $x_{a}$ is the coordinate of the boundary between the hindered and unhindered layer, we get $\bar{p}_{d}-p_{a} \sim x_{a}^{-2 n}$. Substituting $f_{d} d w_{d} \sim x_{a}^{2}$ into eq. (16), we obtain $d=x_{a}^{-2 n-2}$. Now $f_{d} \sim x_{a}^{2}$, $x_{a} \sim \sqrt{\delta_{a}}, \delta_{a} \sim h$; so finally, according to eq. (29), we have

$$
\varphi \sim h^{n-1}
$$

B2. - The layer between two flat plane wedges almost in contact. The thickness of layer is $\delta_{d} \sim x$ where $x=$ distance from the contact line. Now for the unidirectional flow of speed $v$ we have $v \delta_{d} \sim x, \partial p_{d} / \partial x \sim x^{-n}$. By integration $\bar{p}_{d}-p_{a} \sim x_{a}^{-n+1}$ and since $f_{d} d w_{d} \sim x_{a}, f_{d} \sim x_{a}$, $\delta_{a} \sim x_{a}, \delta_{a} \sim h$ we obtain $d \sim x_{a}^{-n}$ and

$$
\varphi \sim h^{n-2}
$$


B3. - The layer between two spheres without contact with a negligible variation of thickness. The thickness is $\delta_{d}=\delta_{o}+\delta_{2} x^{2}$ where $\delta_{2} x^{2} \ll \delta_{o}, x$ denoting the distance from the point of minimum thickness; the radial velocity is $v \sim x$, so that $\partial p_{d} / \partial x \sim x$ and by integration $\bar{p}_{d}-p_{a} \sim x_{a}^{2}$. Since $f_{d} d w_{d} \sim x_{a}^{2}$ we get $d=$ const. and

$$
\varphi \sim f_{d}^{-2}
$$

B4. - The layer between two flat plane wedges without contact, with negligible thickness variation. The velocity $v$ of the unidirectional flow is $v \sim x, x$ is the distance from the centre line and the thickness is $\delta_{d}=\delta_{o}+\delta_{1} x$ (where $\delta_{1} x \ll \delta_{o}$ ). Then $\partial p_{d} / \partial x \sim x$ and by integration $\bar{p}_{d}-p_{a} \sim x_{a}^{2}$. Since $f_{d} d w_{d} \sim x_{a}$ we get $d \sim x_{a} \sim f_{d}$ and

$$
\varphi \sim f_{-}^{-3}
$$

Recalling that approximately $f_{d} \sim h$, we see that the cases B3, B4 yield a decrease of $\varphi$ with $h$, which is the opposite of the cases B1, B2. We believe that variable thickness of the gaps is the statistically dominant case, rather than constant thickness, especially in the vicinity of the thinnest gaps, i.e. for $h \rightarrow 0$. Nevertheless, for $h \rightarrow 1$ the cases B3, B4 may have a greater role, weakening the increase of $\varphi$ with $h$.

\section{APPENDIX C}

\section{Additional comments on concrete deformation}

\section{C1. Cement paste and concrete}

The aggregate in concrete represents a component whose creep and hygrometric deformations are relatively negligible. The fact that besides the elastic particles of hydrated cement and unhydrous cement we have an additional elastic component, the aggregate, requires us to add further springs (or Powers' units) to our model which thus acquires a wider spectrum of retardation times. However, in an even more restricted range of response delay, the three-element model may still be used as a first approximation for concrete.

In water diffusion an additional effect is the exchange of water between aggregate and cement paste. Well saturated aggregate supplies water needed for hydration, while unsaturated aggregate will imbibe water and intensify self-desiccation. Strictly speaking, this is another delayed effect in microscopic local flow. Approximately, we may account for it simply by using proper values of self-desiccation $h_{s}$, depending on the initial saturation of the aggregate.

\section{C2. Separability of creep and shrinkage}

The reason why this question has been an object of discussion for a long time, can be explained by misunderstandings about its meaning.

In one sense it is clear that creep practically always accompanies shrinkage and may not be separated from the calculation of shrinkage since at nonuniform humidity distribution the free shrinkage strains would be generally incompatible.

Physically, the creep of concrete is essentially of the same nature as shrinkage-one is caused by external load, the other by the loading produced by tension in adsorbed films. Mathematically, the separability is to be understood as the principle of superposition. Shrinkage is defined as the deformation for zero surface loads whilst creep (incl. instantaneous deformation) is the remainder. Then, as long as all the equations for the stress and strain problem, i.e. stress-strain law, as well as differential equilibrium and geometric equations with boundary conditions, are linear, the principle of superposition is valid and the responses to various load terms (or absolute terms) are additive. The loading term for shrinkage is $\sigma_{a}$ in eq. (25); the loading terms for " creep " appear in the boundary conditions or equilibrium equations. If the solution of the complete above-mentioned system of equations, in the case of shrinkage (no load) is denoted by $\sigma^{s}, \varepsilon^{s}$ and in the case of creep $\left(\sigma_{a}=0\right)$ by $\sigma^{c}, \varepsilon^{c}$, it would be trivial to demonstrate that the solution in the case of both the $\sigma_{a}-$ term and the loading terms is $\sigma^{s}+\sigma^{c}$ and $\varepsilon^{s}+\varepsilon^{c}$. With respect to some objections in the past, it is worthwhile to stress that for the shrinkage problem and the creep problem the humidity conditions must be the same and the body identical in size and shape. The shrinkage and creep on specimens of different size or shape, or with different humidity distributions in time, may not be added. (Thus, it is no puzzle that the total deformation under load in a certain drying environment is greater than the sum of the deformation whitout load in the same environment plus the deformation under load at $h=1$ ).

\section{C3. Poisson ratio and shear creep}

Since there is no reason why the ratios $\varphi / \psi, G_{b} / K_{b}, G_{c} / K_{c}, G_{d} / K_{d}$ for volumetric and deviatoric creep should be equal, the Poisson ratio is not a material constant, but is time variable, depending on the type of loading, etc. The same has been known about polymers for a long time [64,65]. This agrees with some recent observations [85], indicating that in a standard creep test the Poisson ratio 
decreases with time. This fact suggests that the Poisson ratio corresponding to $G_{c} / K_{c}$ is less than that corresponding to $G_{b} / K_{b}$ (eq. 60). The initial rise of the curves on figure 16 pertains to the assump. tion that the rate of the deviatoric creep (eq. 60) should be higher than that for volume creep. This could be explained by the assumption that the average distance of flow between the compressed and dilated layers in shear creep is shorter than the effective average distance between hindered layers and capillary pores in volume creep.

\section{C4. Tensile creep}

For the elastic as well as dissipative mechanisms described, there cannot be any physical difference between the stresses of opposite sign. The elastic potential and the dissipative potential [63] must vary continuously with stress in the vicinity of an unstressed state. By virtue of this, the tensile and compressive creep (and elastic strain) for sufficiently small stress must be the same and the only justified question is, " above what stress limit does the tensile creep start to be nonlinear (which is due mainly to microcracking)?"

\section{C5. Rate and final value of creep. Bending creep}

The humidity or temperature effect on creep is essentially caused by a change of the creep rate given by $\varphi$ (or retardation times of sorption elements, reflecting the rate of diffusion in the adsorbed layers). It is incorrect to seek it in a change of the "final value" of creep. This is merely an apparent feature. For jnstance, the creep $\varepsilon_{c 1}$ at $h=1$ and $\mathrm{T}=25^{\circ} \mathrm{C}$ for a period of 10 years would be regarded as the final value. Drying environment, or higher $h$ or $\mathrm{T}$ has the same effect as a reduction (contraction) of the time scale (or a proportional reduction of all retardation times in the retardation spectrum). As an example, if $h=0.3$ (or $\mathrm{T}=50^{\circ} \mathrm{C}$ ) the above value $\varepsilon_{c 1}$ would then be obtained in 1 month, say, instead of 10 years, as formerly; and the "final value "for 10 years, say, would now be equal to the value at 1200 years in the former case (if it were measurable). These analogies are, of course, disturbed by the increase of elastic constants $K_{c}$ and $G_{c}$ with age, affecting also the apparent "final value" of creep.

Saying that creep is stress-induced shrinkage is physically admissible but taking it literally has led to misunderstanding about the humidity effect on bending creep. Its explanation was unclear since it was erroneously asserted that drying effect should be added at the compression side of beam and subtracted at the tensile side. This is true, but explains merely that shrinkage is not affected by bending which is clear from linear theory. The drying term for creep, i.e. the term containing $\dot{h}$ in (25) and (37), changes its sign with the stress. This effect, as well as the rate effect mentioned above, is obviously the same for compression and tension, and thus is also the same for bending and torsion. Explaining the effect of drying on bending creep ("Pickett effect" [11]) by some nonlinearity at high stresses induced by shrinkage is at variance with the linearity of creep, and also with the fact that creep increases with $h$ if $h$ inside the sample is constant during creep $[30,31]$.

\section{C6. Temperature dependence of the rate of creep}

If the activation energies for the temperature dependence of $a_{T}, b_{T}, \beta_{T}$ could be assumed equal it would be possible to transform our constitutive equation to a temperature independent form, introducing a new variable $t^{\prime}$ defined by the relationship $t^{\prime}=d t / b_{\mathrm{T}}$. This method is widely utilized for creep of polymers $[64,65,80], t^{\prime}$ is called reduced time and $b_{\mathrm{T}}$ the shift function. In a logarithmic time scale a change of $T$ would then be interpreted by a displacement of the response curve (e.g. creep curve) in the direction of the axis $t[64,65]$.

Above $90^{\circ} \mathrm{C}$ the effect of temperature on the creep rate becomes reversed, [18, 74] and the rate decreases with $\mathrm{T}$. This might be due to physico-chemical changes in cement paste above that temperature $[57,77,78]$. When concrete is allowed to dry with increasing $T$, the creep rate will also change with $h$ and when $h$ approaches zero the creep rate will diminish.

\section{C7. Hygrothermic dilatation and thermal swelling}

Part of delayed thermal dilatation may be explained by the higher coefficient of thermal expansion of water. Then with a rise in temperature, the load-bearing water becomes immediately compressed and then gradually "squeezed out". According to this effect alone, however, the delayed effect and immediate dilatation would have to diminish with decreasing $h$. This is not observed. In effect, down to about $h=0.7$ an increase takes place $[68-70,2]$. This increase can be accounted for only by hygrothermic dilatation which is a consequence of the hygrothermic coefficient $x$ whose value tends to 0 as $h \rightarrow 1$. 
It is of interest to integrate eq. (31), including the term $(50)$, for a constant rate $\dot{T}$, assuming the material initially $(t=0)$ at rest. For a nearly uniform distribution of $T$ (small sample or slow heating) and uniform $h$ (sealed sample) the result is $(\sigma=0)$ :

$$
\begin{aligned}
\varepsilon=\alpha_{o} \mathbf{T} t & +\left(\alpha_{o}-\alpha_{1}\right) \frac{\dot{\mathrm{T}}}{\varphi}\left(1-e^{-\varphi t}\right) \\
& +\dot{\sigma}_{a}\left[\frac{1}{\mathbf{K}_{c}}\left(\frac{1}{1+\mathbf{K}_{c} / \mathbf{K}_{a}}+\frac{f_{d}}{f_{a}}\right)-\frac{1}{\mathbf{K}}\right] t+\sigma_{a} \frac{1}{\varphi \mathbf{K}_{c}}\left(\frac{1}{1+\mathbf{K}_{c} / \mathbf{K}_{a}}+\frac{f_{d}}{f_{a}}\right)\left(1-e^{-\varphi t}\right)+\varepsilon^{\prime}
\end{aligned}
$$

where $\varepsilon^{\prime}=$ an additional term, accounting for the variation of $\dot{\sigma}_{a}, \phi, \alpha_{1}, \alpha_{0}$ with T, which tends to zero (as $t^{2}$ ) for small temperature change. The third and fourth terms express the instantaneous and delayed thermal swelling. Calculating $\dot{\sigma}_{a}$ from $(20)$ we see that depends on $\dot{\sigma}_{a} \varkappa$ (and T, $\dot{\mathrm{T}}$ ).

\section{C8. Effect of capillary water and chemically bound water}

Imagine that in figure 1 the adsorbed layers do not come into contact. Then the pressure on solid surfaces is developed only by capillary water filling the long gap. In equilibrium this pressure, $p_{c}$, is expressed by eqs. (2a) and (1). Sudden application of load will destroy this condition of thermodynamic equilibrium, and the difference $p_{c}-p_{a}$ will cause the capillary water to flow slowly out towards the surface meniscus. Similarity with the behavior of hindered adsorbed water is thus clear.

Note. With respect to our assumption of small deformation it is impossible to explain creep by a change of the curvature radius of surface meniscus, corresponding to the change of distance between the contact points of the meniscus at the solid surfaces.

The distinction between various forms of fixed water is defined only by the value of the binding energy and is not clear cut. Some water molecules chemically bound in cement crystals, such as the interlayer hydrate, may have a lower binding energy than some adsorbed molecules [81, 48]. Therefore they form part of evaporable water, $w_{e}$. Their equilibrium with other forms of water is again expressed by equality of partial free energies (chemical potentials) $G^{1}$. Because $G^{1}$ depends on pressure (as in eq. A1, A9), an application of load, causing pressure increase in the interlayer hydrate, will destroy this equality and the created difference in $\mathrm{G}^{1}$ will cause a gradual diffusion [71] of the interlayer hydrate out of (or into) the hydrated cement and allow a gradual contraction (dilation) of the space left (entered), until pressure is readjusted and new equilibrium reached.

Obviously, the described mechanisms for both the chemically bound and capillary water are phenomenologically similar to our mechanism for adsorbed water, so that formally same equations as $(17),(25),(26),(32 d),(38),(39)$ would be obtained. We can thus conclude that the resulting macroscopic constitutive equation is the same as for microscopic diffusion of any form of load-bearing water, whether it is adsorbed, or chemically bound, or capillary. (Of course, from the physical viewpoint the distinction between them is an important object of discussion.) Simultaneous effect of all these states of water means that we should add more units in the chain on figure $5 b$, getting a wider relaxation spectrum. What is finally important in our model is not which state of water has the dominant role but only that there exist a sufficiently large quantity of oriented layers (or tiny tubes) of water which can withstand static stress and respond by diffusion to the disruptions of its thermodynamic equilibrium.

\section{C9. Effect of volume changes at hydration}

Cement hydration is caused by a difference in partial free energies $G^{1}$ (chemical potentials) between unhydrous cement, and hydrated cement. Since $G^{1}$ depends on $p$ (eq. A1), application of a pressure on some part of hydrated cement may create equilibrium and stop further hydration (and the inherent growth in volume) in that part. Conversely, if growth in volume is opposed by the existing structure, a pressure needed for equilibrium will be developed. This pressure is certainly one cause why some (not all) cement pastes swell if immersed in water from the time of casting.

\section{C10. Dissolution under load and recrystallization}

If a pressure is applied on a part of hydrated cement already in equilibrium, the inherent change in $G^{1}$ will cause an opposite process than in 69 , i.e. a dehydratation, or dissolution of the imperfect crystalline particles of cement gel in that part. This will be accompanied by diffusion of matter into other pores where pressure is not active, and recrystallization [86] of imperfect crystallites of cement gel in them. This recrystallized matter will somewhat increase the resistance of the solid framework at a subsequent change of load, and thus cause nonlinearity with respect to stress, and irreversibility. This nonlinearity, however, will be small and normally undetectabte since the volume of the dissolved and recrystallized matter must be smaller than the volume change of porous material (or smaller than the volume $e_{11} \times 1 \times 1$ at the strain deviator of form (35)) which itself is negligibly small (assumption 2) with respect to the total volume of the solid per unit volume of porous material. If a tension is applied instead of a pressure, the reverse mechanism occurs-matter will be dissolved in the pores where this tension is not active, then diffuse into the thin gaps under tension 
and recrystallize in them. This mechanism will lead again to the same form of constitutive equation as eqs. (25), (26), (37), and will be represented by still another unit in the chain on figure $5 b$, eq. $(32 d)$. If the compressive volume creep after rewetting of a predried sample is found to be greater than the expansion obtained at rewetting [31] plus the elastic deformation of a dried sample, then a plausible explanation would be provided by this mechanism. It is still questionable at present whether this mechanism can really be important but the enormous internal surface and the smallness of the volume to be dissolved speak for it even if the solubility is very low.

Note. Nevertheless, even a small amount of recrystallized matter may cause some nonlinearity with respect to stress if it recrystallizes just at the boundary of hindered layers where the gaps are so thin that a large area may be covered by it. Perhaps this is the reason why, after a longer period of creep under sustained load, the instantaneous modulus for a subsequent load increment seems to be greater than for unloading [96] or for the unloaded sample [97] (by about $10 \%$ ).

\section{C11. Slide movements and cement gel viscosity in creep}

It may be admitted that besides contraction of hindered layers, slip movements between opposite solid surfaces could exist [87]. This applies for deviatoric, and in some extent even for volumetric strains, as it is visualized for the two configurations on figure 7 . At a change of thickness of a hindered layer, the water molecules are forced to travel a distance which is great with respect to its thickness, whereas at slide movement the molecules travel a distance which is small with respect to the thickness of layer. Realizing this, we are tempted to conclude that the resistance to sliding should be negligible if compared with the resistance to the change of thickness of these layers.

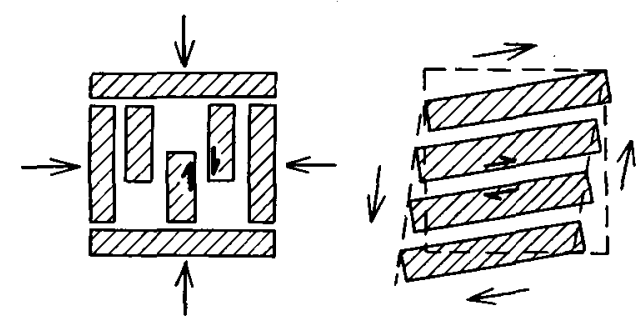

FIG. 7. - Examples of slip movements between particles. (In the gaps where slip is indicated there is assumed to be no solid connection, i.e. sufficient thickness of the gap.)

This mechanism alone would be able to account for the fact that creep is lower for a smaller $w$ or $h$, as the "water lubrication" effect gradually disappears with $w$, but probably not for the increase of creep due to drying (during creep). Nevertheless, at present it could hardly be excluded that this mechanism might be operative simultaneously with microscopic diffusion (see also [52]) which might be expressed by some additional units in the chain model in figure $5 b$, containing dashpots rather than sorption elements. The corresponding equations in the system $(32 d)$ would be characterized by $\sigma_{a d_{i}}=0$ (or $f_{d_{i}}=0$ ) and $K_{h_{l}} \rightarrow \infty, \mathrm{G}_{h_{i}} \rightarrow \infty$.

\section{C12. Microcracking}

Formation of a microcrack is accompanied by reduction of the macroscopic average elastic moduli. Therefore, microcracking is necessarily linked with nonlinearity with respect to stress and strain. Thus it has no place in a theory which is linear but it is certainly the main cause of nonlinear creep (and irreversibility at high stresses). In effect, microcracking has been proved really significant only above 0.5 or 0.4 of the ultimate stress. Anyhow, it is dubious whether microcracking alone could account for the various effects of humidity.

A special property of cement paste is the ability to heal closed cracks, i.e. restore continuity (by recrystallization or formation of bond) [93], [95]. A sequence of shear cracking and subsequent healing may be a part of the creep mechanism which will not disturb linearity with respect to stress. It would be formally equivalent to the sliding mechanism mentioned in C11 (or identical if the cracks are slides between particles).

\section{C13. Strain gradient effect}

The difference between bending and axial creep has been sometimes attributed to the presence of strain or stress gradient. In the example of figure 14, however, this effect arises only because of a different correspondence between the stress and humidity distributions. Stress gradient as such cannot appear in our form of equations. Strain gradient is linked with the couple stresses [62] and these may carry some part of the bending moment, provided that the beam depth is sufficiently small with respect to the aggregate size. 


\section{C14. Autogenous shrinkage}

The shrinkage of a sealed sample certainly depends on the amount of self-desiccation, just as drying shrinkage depends on decrease of internal humidity. An indirect effect of self-desiccation as a humidity change on the rate of hydration and growth of cement gel is also involved.

\section{C15. Shrinkage due to solid surface tension}

Solid particles become compressed because of their surface tension, depending on humidity [32, $38,88]$. This compression results form the static equilibrium condition and follows the humidity change immediately. Therefore, this effect is formally identical with our immediate component of shrinkage introduced by the term $\sigma_{a}$ in eqs. (25), (26).

\section{C16. Intrinsic influences}

The effect of water-cement ratio, kind and percentage of aggregate and cement, etc. [15, 14], are all conditions fixed once forever when the material is cast. They do not affect the form of our equations but only the values of their coefficients. We need to know them for an efficient design of mix and prediction of creep but we should be able to analyze the stresses and strains without any knowledge about them if we had data about a sufficient series of tests on the one specific concrete of our structure. These influences represent an important separate problem which is beyond the scope of this paper.

\section{C17. Heat conduction and temperature distribution}

Prior to the stress and strain analysis, T must be determined as a function of $t, x, y, z$. Similarly as for water diffusion, according to our assumptions this temperature problem and the stress and strain problem are uncoupled, i.e. deformation does not affect $\mathbf{T}$. However, the temperature problem is coupled with the water diffusion problem because the speed of water flow depends on $T$, the heat conductivity depends on water content [14], and the heat of hydration, heat of evaporation at the surface and heat of adsorption (which could reach $20 \mathrm{cal} / \mathrm{g}$ of cement) depend on humidities and their change. This coupling becomes especially important when considering fire exposure.

\section{APPENDIX D}

\section{Scheme of program for stress and strain analysis}

For each time step $\Delta t$ the procedure is as follows.

1 Save the initial values in the step of $\sigma_{,} s_{i j}, \sigma_{d}, s_{d_{i j}}, t_{e}, h, \mathrm{~T}\left(\sigma^{*} \leftarrow \sigma\right.$ etc.) for all nodes (elements).

2 Using $t_{e}$ and $h$, calculate $K_{b}, K_{c}, K_{d}, G_{b}, G_{c}, G_{d}$ (or $K_{c}^{\prime}, G_{c}^{\prime}$ if (53) valid). Then compute $K$, G. Repeat for all nodes.

3 Using $h, \Delta h, \mathrm{~T}, \Delta \mathrm{T}$, calculate $\Delta \sigma_{a}, \sigma_{a d}, \alpha_{1}, \Delta \mathrm{T}, a_{\mathrm{T}}, \varphi, \psi$ and $\varepsilon^{0}, e_{i j}^{0}$ (eqs. 56, 57) for all nodes.

4 Solving the elasticity problem with $\varepsilon^{0}, e_{i j}^{0}$ as prescribed initial strains, and given changes of loads or prescribed displacements, determine $\Delta \varepsilon, \Delta e_{i j}, \Delta \sigma, \Delta s_{i j}$ for all nodes.

5 Using $h, \Delta h, \mathrm{~T}, \Delta \mathrm{T}$, calculate again $\Delta \sigma_{a}$ (saves storage), $\alpha_{o} \Delta \mathrm{T}$. Then calculate $\Delta \sigma_{d}, \Delta s_{d_{i j}}$ from eqs. (58), (59). Repeat for all nodes.

6 Calculate $\beta_{\mathrm{T}}, \Delta t_{e}, k, c, b_{\mathrm{T}}, \Delta h_{\mathrm{d}}, \chi, \Delta h^{1}$ (or $k^{\prime}, x^{\prime}$ if $\Delta h>0$ ) for all nodes.

7 Calculate $\Delta h$ and $\Delta \mathrm{T}$ for all nodes (finite difference form of eq. 8).

8 Assign $\sigma \leftarrow \sigma^{*}+\Delta \sigma / 2, \ldots, h \leftarrow h^{*}+\Delta h / 2$ to all variables (but $\varepsilon, e_{i j}$ ), for all nodes.

9 Return to 2 and proceed again until 8 , with several repetitions (iterating thus the mean values of $\sigma, h, \ldots$ in the step $\Delta t$ ).

10 Compute initial values for the next step of $\sigma, s_{i j}, \varepsilon, e_{i j}, \sigma_{d}, s_{d_{i j}}, t_{e}, h, \mathrm{~T}$, assigning $\sigma \leftarrow \sigma^{*}$ $+\Delta \sigma, \ldots, \sigma_{d} \leftarrow \sigma_{d}^{*}+\Delta \sigma_{d}, \ldots t_{e} \leftarrow t_{e}^{*}+\Delta t_{e}, \ldots \varepsilon \leftarrow \varepsilon^{*}+\Delta \varepsilon, \ldots$ Print these values. Assign $t \leftarrow t+\Delta t$ and go to 1 (next step) if $t<$ final time.

We see that in comparison with the elasticity problems we have to store for all nodes the additional values $\sigma_{d}, s_{d_{i j}}, t_{e}, h, \mathrm{~T}, \Delta h, \Delta \mathrm{T}, \Delta \sigma, \Delta s_{i j}$. (Storage space for $\Delta \sigma, \Delta s_{i j}$ could be equivalenced with $\sigma, s_{i j}$; instead of storing $K, G$, these values could be recalculated whenever needed.) If we use the model on figure $5 b$, eq. (32d), we must compute and store $\sigma_{d_{1}}, \sigma_{d_{2}}, s_{d i_{j 1}}, s_{d i_{j 2}}$ for all nodes.

In our example of prisms, because of the uniaxial state of stress only the values $\sigma, s_{23}$ need to be considered in all nodes throughout the thickness of the cross section since the axial normal stress is $\sigma_{11}=3 \sigma$ and $s_{11}=2 \sigma, s_{22}=-\sigma, s_{33}=s_{12}=s_{13}=0, x_{1}=x$ being the longitudinal axis of prism, $x_{2}=y$ the axis across the thickness (in the direction of flow). The step 4 of the above scheme is here as simple as follows. 
4.1. Calculate $\sigma^{0} \leftarrow \mathrm{K} \varepsilon^{0}, s_{11}^{0} \leftarrow 2 \mathrm{G} e_{11}^{0}$ for all nodes.

4.2. Calculate Young modulus $\mathrm{E}$ for all nodes.

4.3. Compute axial, bending and torsional rigidities $R_{1}, R_{2}, R_{3}$ of the cross section.

4.4. Calculate the resultants of the axial normal stresses $\sigma_{11}^{0}=\sigma^{0}+s_{11}^{0}$ over the cross section, i.e. axial force $\mathbf{P}^{0}$, bending moment $\mathbf{M}^{0}$, torque $\mathbf{T}^{0}$.

4.5. Add to $\mathrm{P}^{0}, \mathrm{M}^{0}, \mathrm{~T}^{0}$ the given changes of applied loads.

4.6. Calculate $\Delta \sigma \leftarrow \mathrm{E}\left(\mathrm{P}^{0} / \mathbf{R}_{1}+\mathrm{M}^{0} \boldsymbol{y} / \mathbf{R}_{2}\right) / 3, \Delta s_{23} \leftarrow \mathrm{G} \mathrm{T}^{0} y / \mathbf{R}_{3}, \Delta \varepsilon \leftarrow \Delta \sigma / \mathrm{K}, \Delta e_{23} \leftarrow \Delta s_{23} / 2 \mathrm{G}$ for all nodes.

4.7. Assign $\Delta \sigma \leftarrow \Delta \sigma+\sigma^{0}, \Delta s_{23} \leftarrow \Delta s_{23}+s_{23}^{0}$ in all nodes.

In case of a cylinder we have to consider also the non-zero circumferential normal stress $s_{33}+\sigma$, as well as the radial stress $s_{22}+\sigma$.

\section{AC KNOWLEDGEMENTS}

The greatest part of the present research was carried out during the author's leave of absence from the Building Research Institute, Technical University, Prague, on a 15 months visiting appointment in $1967 / 8$ as a Senior Research Fellow at the University of Toronto, Department of Civil Engineering, under the sponsorship of Ford Science Foundation. Grateful appreciation is due to Professor M. W. Huggins who supervised the research. The author is greatly indebted to Visiting Professor T. C. Powers for his numerous valuable critical remarks on the original draft of this study and for correction of several erroneous statements, as well as for consultations during the author's attendance of his seminar on "Physical Properties of Cement Paste".

The first impulse to try the present approach was given to the author by Director of Research R. L'Hermite, F.N.R.B.T.P., Paris, during the author's 6 months stay in 1966/7, on a grant of A.S.T.E.F. The author wishes also to thank Professors B. Bresler, M. Polivka and D. Pirtz, and Visiting Professor G. Winter at the University of California, Berkeley, Department of Civil Engineering, for their discussion on the subject during the author's visiting appointment as Associate Research Engineer in 1969. The discussions with Assistant Professor J. Timusk, as well as consultations on programming with Associate Professor R. A. Collins at the University of Toronto are deeply appreciated too. The computer facilities (IBM 7094) were rendered by the Institute of Computer Science, University of Toronto.

\section{REFERENCES}

[1] L'Hermite, R. - Les déformations du béton, Cahier de la Recherche no $^{12}$, I.T.B.T.P. et A.F.R.E.M.C., Paris, Eyrolles 1961.

[2] L'Hermite, R. - Volume changes of concrete, in "Chemistry of cement", 4th Intern. Symp., Wash. D.C., 2-7 Oct. 1960, U.S. Nat. Bureau of Standards, Monograph 43, Vol. II, Paper V-3, 659-694, 1962.

[3] L'Hermite, R., Mamillan R., et Lefèvre, C. Nouveaux résultats de recherches sur la déformation et la rupture du béton, Annales de l'Inst. Techn. du Bât. et des Travaux Publ., no 207-8, 1965, 323-360.

[4] L'Hermite, R., and Mamillan M. - Further results of shrinkage and creep tests, Intern. Conf. on the Structure of Concrete, Paper H2, 1965, Imperial College, London, publ. by Cement and Concrete Assoc.

[5] KeSLER, C. E. and ALI, J. - Rheology of concrete, $A$ review, Univ. of Illinois, Dept. Theor. Appl. Mech., Report No. 636, Urbana 1963.

[6] Wallo, E. U. and Kesler, C. E. - Prediction of creep in structural concrete, Dept. of Theor. and Appl. Mech., Rep. No. 670, Univ. of Illinois, Urbana, Dec. 1966.
[7] BAžANT, Z. P. - Creep of concrete and structural analysis (in Czech), SNTL (State Publ. House of Tech. Lit.), Prague, 1966.

[8] Gvozpev, A. A. - Creep of concrete (survey, in Russian), in "Mekhanika Tverdogo Tela", Proc. of the 2nd Nat. Conf. on Theor. and Appl. Mech., Acad. Sci. USSR, Moscow 1966, 137-152.

[9] W WGNER, O. - Das Kriechen unbewehrten Betons, Deutscher Ausschuss für Stahlbeton, H. 131, W. Ernst und Sohn, Berlin, 1958.

[10] Hansen, T. C. and Mattock, A. H. - Influence of size and shape of member on the shrinkage and creep of concrete, J. ACI, 63, 267-290, 1966.

[11] Picketr, G. - The effect of change in moisture content on the creep of concrete under a sustained load, Journal ACI, Feb. 1942, 333-355.

[12] Ross, A. D., Illston, J. M. and England, G. L. - Short- and long-term deformations of concrete as influenced by its physical structure and state, Intern. Conf. on the Structure of Concrete, Paper H1, 1965, Imperial College, London, publ. by Cement and Concrete Assoc.

[13] Sackman J. L. - Creep in concrete and concrete structures (survey), Princeton University Conf. on Solid Mech., Nov. 21-23, 1963, Princeton, N.J. 
[14] Neville, A. M. - Properties of concrete, J. Wiley, New York 1963.

[15] Troxell, G. E., Davis, H. E. and Kelly, J. W. - Composition and properties of concrete, $2 \mathrm{nd}$ pr., McGraw Hill, New York 1968.

[16] AlI, J. and Kesler, C. E. - Creep in concrete with and without exchange of moisture with the environment, Univ. of Illinois, Dept. Theor. Appl. Mech., Report No. 641, Urbana, Dec. 1963.

[17] Wallo, E. U., Yuan R. L. and Kesler, C. E. Free shrinkage of concrete and mortar, Dept. of Theor. and Appl. Mech., Rep. No. 670, Univ. of Illinois, Urbana, Dec. 1966.

[18] Browne, R. D. - Properties of concrete in reactor vessels, Conf. on Prestressed Concrete Reactor Pressure Vessels, Institution of Civil Engineers, Paper C13, London, March 1967.

[19] Ulickiy, T. T. et al. - Analysis of reinforced concrete structures with respect to long-time processes (in Russian), Gosstroyizdat, Kiev 1960.

[20] Рroкороvich, J. E. - Effect of long-time processes on the state of stress and strain in structures (in Russian), Gosstroyizdat, Moscow 1963.

[21] Aroutiounian, N. Kh. - Applications de la théorie du fluage, Eyrolles, Paris 1957.

[22] BažAnt, Z. P. - Phenomenological theories for creep of concrete based on rheological models, Acta Technica CSAV (Prague), 1966, No. 1, 82-109.

[23] BAZ̆ANT, Z. P. - Electric analogues for creep of concrete structures, Conf. on Exper. Methods of Investigating Stress and Strain in Structures, Techn. Univ. Prague, Building Res. Inst., 58 Oct. 1965, 797-809.

[24] McHeNry, D. - A new aspect of creep in concrete and its application to design, Proc. ASTM, Vol. 43, 1943, 1069-1087 (with discussion by H. Laedre$\operatorname{man})$.

[25] Bresler, B. and Selna, L. - Analysis of time dependent behavior of reinforced concrete structures, in "Symposium on Creep", Amer. Concrete Inst., Publ. SP-9, Detroit 1964, 115-128.

[26] Bažant, Z. P. - Die Berechnung des Kriechens und Schwindens nicht-homogener Betonkonstruktionen, 7th Congress, Intern. Assoc. for Bridge and Struct. Engng., Prelim. Publ., Rio de Janeiro 1964, 10-16 Aug., 887-897.

[27] BAžANT, Z. P. - Creep stability and buckling strength of concrete columns, Magazine of Concrete Research, Vol. 20, No. 63, June 1968, 85-94.

[28] Mullen, W. G. and Dolch, W. L. - Creep of Portland cement paste, Proc. ASTM, 64, 1964, 1146-1170.

[29] GlückLich, J. and Ishai, O. - Creep mechanism in cement mortar, Jour., Amer. Concrete Inst., Vol. 59, 1962, 923-948.

[30] Cilosani, Z. N. - On the true mechanism of creep of concrete (in Russian), Beton i Zhelozobeton (Moscow), No. 2, 1964, 75-78.

[31] Hannant, D. J. - The mechanism of creep of concrete, Materials and Structures (Bulletin RILEM), No. 5, 1968, 403-410.

[32] Powers, T. C. - Mechanism of shrinkage and reversible creep of hardened cement paste, International Conf. on the Structure of Concrete, London, Sept. 1965, paper G1, Imperial College, publ. by Cement and Concrete Assoc.
[33] Powers, T. C. - The nature of concrete, in "Significance of Tests and Properties of Concrete and Concrete-making Materials, ASTM, STP No. 169-A, 61-62, 1966 (P.C.A. Bulletin No. 196).

[34] Powers, T. C. and Brownyard, T. C. - Studies of the physical properties of hardened Portland cement paste, Journal of the Amer. Concr. Inst., 1946, pp. 101-132, 249-336, 469-504, 1947, pp. 549-602, 669-712, 845-880, 933-992 (P.C.A. Bulletin, No. 22).

[35] Lynam, C. G. - Growth and movements in Portland cement concrete, Oxford Univ. Press, London 1934.

[36] Powers, T. C. - Some observations on the interpretation of creep data, Bulletin RILEM (Paris), No. 33, Dec. 1966, 381-391.

[37] Hrennikof, A. - Shrinkage, swelling and creep in cement, Proc. ASCE, J. of Eng. Mech. Div., EM3, July 1959, 111-135.

[38] Powers, T. C. - The thermodynamics of volume change and creep, Materials and Structures (Bulletin RILEM), No. 6, Nov.-Dec. 1968, 487507.

[39] Biot, M. A. - Mechanics of deformation and acoustic propagation in porous media, Journal of Applied Physics, Vol. 33, No. 4, pp. 1482-1498.

[40] Mills, R. H. - Influence of water in areas of restricted adsorption on properties of concrete, Materials and Structures RILEM, Vol. 1, No. 6 , Nov.-Dec. 1968, 553-558.

[41] Ross, S. and Olivier, J. P. - On physical adsorption. Interscience Publishers, New York 1964.

[42] Brunauer, S. - The adsorption of gases and vapors, Vol. 1, Prinoeton Univ. Press, 1943.

[43] Brunauer, S., Emmett, P. H. and Teller, E. Adsorption of gases in multimolecular layers, J. Amer. Chemical Soc., Vol. 60, 1938, 309-319.

[44] Guggenheim, E. A. - Thermodynamics, classical and statistical, in Encyclopedia of Physics, ed. by S. Flügge, Vol. III $/ 2$, Principles of thermodynamics and statistics, Springer, Berlin 1959.

[45] Coнan, L. H. - Hysteresis and the capillary theory of adsorption of vapors, J. Amer. Chemical Soc., Vol. 66, 1944, 98-105.

[46] Powers, T. C. - $A$ discussion of cement hydration in relation to the curing of concrete, Proc. of the Highway Research Board, 27, 1947, 178-198 (P.C.A. Bulletin No. 25).

[47] Shuttleworth, R. and Bailey, G. L. J. Spreading of a liquid over a rough solid, in "Interaction of water in porous solids ", Discussions of the Faraday Society, No. 3, 1948, Gurney and Jackson, London, 16-28.

[48] Ha Rмathy, T. Z. - Moisture sorption of building materials, Technical paper No. 24,2, Div. Building Research, National Research Council, Ottawa, March 1967.

[49] Powers, T. C. - Some physical aspects of the hydration of Portland cement, Journal of the Portland Cement Assoc. Research and Dev. Lab., Vol. 3, Jan. 1961, No. 1, 47-56.

[50] Aleksandrovs KiI, S. V. - Analysis of plain and reinforced concrete structures for temperature and moisture effects (with account of creep) (in Russian), Stroyizdat, Moscow 1966. 
[51] Copeland, L. E. and Bragg, R. H. - Self desiccation in Portland cement pastes, Proc., Amer. Soc. for Testing Materials, No. 204, Feb. 1955 (P.C.A. Bulletin 52).

[52] Freudenthal, A. M. and Roll, F. - Creep and creep recovery of concrete under high compressive stress, J. Amer. Concrete Inst., No. 12, June 1958, 1111-1142.

[53] Powers, T. C., Copeland, L. E. and Mann, H. M. - Capillary continuity and discontinuity in cement paste, J. of the Portland Cement Assoc. Res. and Dev. Lab., Vol. 1, No. 2, May 1959, 38-48 (P.C.A. Bulletin 110).

[54] BARRER, F. - Diffusion in and through solids, Cambridge University Press, Cambridge 1941 (Chpt. VIII, Migration in the surface layer of solids).

[55] Harmathy, T. Z. - Simultaneous moisture and heat transfer in porous systems with particular reference to drying, Div. of Building Research, National Research Council, Ottawa 1968 (to appear in "Industrial and Engng. Chemistry").

[56] Hanson, J. A. - Effects of curing and drying environments on splitting and tensile strength of concrete, Jour., Amer. Concrete Inst., Vol. 65, July 1968, 535-543 (P.C.A. Bulletin D141).

[57] Abrams, M. S. and Orals, D. L. - Concrete drying methods and their effect on fire resistance, in "Moisture of Materials in Relation to Fire Tests, STP No. $385,1965,52-73$, publ. by Amer. Soc. for Testing Materials (P.C.A. Bulletin 181).

[58] Powers, T. C., Mann, H. M. and Copeland, L. E. - The flow of water in hardened Portland cement paste, Highway Research Board, Report 40, July 1959 (P.C.A. Bulletin 106).

[59] Powers, T. C., Copeland, L. E., Hayes, J. C. and Mann, H. M. - Permeability of Portland cement paste, Journal, Amer. Concrete Inst., Vol. 51, Nov. 1954, 285-298 (P.C.A. Bulletin 53).

[60] Helmuth, R. A. and Turk, D. H. - Elastic moduli of hardened Portland cement paste and tricalcium silicate pastes : Effect of porosity, Symposium on Structure of Portland Cement Paste and Concrete, Highway Res. Board, Spec. Rep. No. 90, 135-144, Washington 1966 (P.C.A. Bulletin No. 210).

[61] Pirtz, D. - Creep characteristics of mass concrete for Dworshak Dam, Rep. No. 65-2, Struct. Engng. Lab., Univ. of California, Berkeley, Oct. 1968.

[62] Eringen, A. C. - Nonlinear theory of continuous media, McGraw-Hill, New York 1962 (Chpt. 5, $8,10)$.

[63] Вıот, M. A. -- Variational principles of irreversible thermodynamics with application to viscoelasticity, Physical Review 97, 1463-1469, 1955.

[64] FeRRY, J. D. - Viscoelastic properties of polymers, New York, 1961.

[65] Williams, M. L. - The structural analysis of viscoelastic materials, AIAA Summer Meeting, Los Angeles 1963.

[66] Wu, Y. C. and Copeland, L. E. - Thermodynamics of adsorption. Barium sulphate-water systems. "Solid surfaces and the gas solid interface ", Advances in Chemistry Series, Amer. Chem. Soc., 1961 (P.C.A. Bulletin 141).
[67] Monfore, G. E. - Private communication, letter of 13 Jan., 1969, Portland Cement Assoc. Res. and Dev. Lab., Skokie, Illinois.

[68] Vironnaud, L. et Van Tanh, N. - Dilatomètre à trépied optique, mesures et résultats sur le béton, Annales Inst. Techn. Bâtiment Trav. Publ., Juin 1954, 521-540.

[69] Meyers, S. L. - Thermal expansion characteristics of hardened cement paste and concrete, Proc., Highway Research Board, Vol. 30, 1950, 193-203.

[70] Heцmuth, R. A. - Dimensional changes of hardened Portland cement pastes caused by temperature changes, Proc. of the Highway Research Board, 40, 315-336, 1957 (P.C.A. Bulletin No. 129).

[71] Brophy, J. M., Rose, R. M. and Wulff J. The thermodynamics of structure, Vol. II of " The structure and properties of materials", ed. by Wulff, J. Wiley, New York 1964.

[72] Glasstone, S., Laidler, K. J. and Eyring, H. - The theory of rate processes, Mc Graw Hill, New York, 1941.

[73] Polivka, M. and Best, C. H. - Investigation of the problem of creep in concrete by Dorn's method, 39th Annual Meeting of the Highway Research Board, Wash. D. C., Jan. 1960.

[74] Nasser, K. W. and Neville, A. M. - Creep of old concrete at normal and elevated temperatures, J. ACI, V. 64, Feb. 1967, 97-103.

[75] Cruz, C. R. - Elastic properties of concrete at high temperatures, Journal of the Portland Cement Assoc. Res. and Dev. Lab., Vol. 8, Jan. 1966, No. 1, 37-45 (P.C.A. Bulletin 191) (with reference 3).

[76] TAYLoR, H. F. V. - The steam curing of Portland cement products, in " Chemistry of Cements", ed. by Taylor, Vol. 1, Academic Press 1964, London, 417-432.

[77] Czernin, W. - Zementchemie für Bauingenieure, Bauverlag, Wiesbaden 1960 (Engl. ed., Cement chemistry and physics for civil engineers, Chemical Publishing Co., New York 1962).

[78] Copeland, L. E., Kantro, D. L. and Verbeck, G. - Chemistry of hydration of Portland cement, in "Chemistry of cement", Proc., 4th Intern. Symp., Wash. D. C., 1960, Nat. Bureau of Standards, Monograph 43, Vol. I, Paper IV-3, 429-465 (P.C.A. Bulletin 153).

[79] VAN VLACK, L. H. - Elements of materials science, Addison-Wesley, Reading, Mass., 1959 (chpt. 8-2).

[80] Bažant, Z. P. - Mathematische Ermittlung der rheologischen Eigenschaften von glassfaserverstärkten Plasten, Proc., Erste Internationale Tagung über GFP und Epoxydharze, Berlin, Institut für Kunststoffe, Deutsche Akademie der Wissenschaften, 23-27 Sept. 1965, Paper E3/1.

[81] Feldman, R. F. and Sereda, P. J. - A model for hydrated Portland cement paste as deduced from sorption-length change and mechanical properties, Materials and Structures (Bulletin RILEM), No. 6, 1968, 509-520.

[82] BažANT, Z. P. - Langzeitige Durchbiegungen von Spannbetonbrücken infolge Schwingkriechens unter Verkehrslasten, Beton und Stahlbetonbau, 1969, No. 12, 282-285. 
[83] BAžANT, Z. P. - Linear creep problems solved by a succession of generalized thermoelasticity problems, Acta Technica CSAV (Prague), 1967, No. 5, 581-594.

[84] Ter HaAr, D. and Wergeland, H. - Elements of thermodynamics, Addison-Wesley, London 1966.

[85] Trmosк, J. - Private oral communication on tests at the University of London by J. W. H. King and Timusk, May 1968, Toronto.

[86] Slate, F. O. and Meyers, B. L. - Some physical processes involved in creep of concrete, to be presented at the "Intern. Conf. on Structure, Solid Mech. and Eng. Design in Civil Engng. Materials", University of Southampton, April 1969.

[87] Ruetz, W. - The two different physical mechanisms of creep of concrete, Intern. Conf. on the Structure of Concrete, Paper C2, 1965, Imperial College, London, publ. by Cement and Concr. Assoc.

[88] Milus, R. H. - Effects of sorbed water on dimension, compressive strength and swelling pressure of hardened cement paste, Symposium on structure of Portland cement paste and concrete, Highway Research Board, Special Report 90, Wash. D. C. 1960, 84-111.

[89] Brunauer, S. - Some aspects of the physics and chemistry of cement, in "The Science of Engineering Materials", ed. by J. E. Goldman, J. Wiley, New York 1957 (P.C.A. Bulletin No. 80).

[90] Powers, T. C. - Hydraulic pressure in concrete, Proc. ASCE, Paper 742, Vol. 81, July 1955, p. 742-5 (PCA Bulletin 63) and experiments by Carlson and Davis in "Final Report of the Subcommittee on Uplift in Masonry Dams", Committee on Masonry Dams, ASCE, Separate $n^{0} 133$, 1952.

[91] Hannant, D. J. - Strain behaviour of concrete up to $95^{\circ} \mathrm{C}$ under compressive stresses, Intern. Conf. on the Structure of Concrete, London, Sept. 1965, Paper C17, Imperial College, publ. by Cement and Concrete Assoc.

[92] Freyssinet, E. - Études sur les déformations lentes des ciments ou retraits, Ier Congrès Inter- national du Béton et du Bêton Armé, ,520-532, Liège 1930, Éditions « La Technique des Travaux », 1932.

[93] Lauer, R. K., Slate, F. J. - Autogenous healing of cement paste, Jour., Amer. Concrete Institute, Proc. V. 52, no 10, June 1956, 10831098 (Disc. p. 1457).

[94] Powers, T. C. - Properties of fresh concrete, New York 1968.

[95] Freyssinet, E. - The deformation of concrete, Magazine of Concrete Research (London), Dec. 1951, 49-56.

[96] Kimishima, H., Kitahara, Y. - Creep and creep recovery of mass concrete, Techn. Report C64001, Sept. 1964, Central Res. Inst. of Electric Power Industry, Tokyo.

[97] Pirtz, D. - Private oral communication, March 1969, University of California, Berkeley.

[98] Sh Kerbelis, K. K. - Influence of combined loading on concrete deformation (in Russian), Issledovania po Betonu i Zhelezobetonu (Riga, ANLSSR), Vol. III, 1958, 56-60.

[99] BAZANT, Z. P. - Influence of creep and shrinkage in statically indeterminate structures of concrete of variable age, Inženýrské Stavby (Prague), 1961, 426-432 (in Czech).

[100]SH KERBELIS, K. K. - On relation of concrete deformation to loading rate (in Russian), Issledovania po Betonu i Zhelezobetonu (Riga, ANLSSR), Vol. III, 1958, 39-55.

[101]Davis, R. E., Davis, H. E., Hamilton, J. S. Plastic flow of concrete under sustained stress, Proc., Amer. Soc. for Testing Materials, Vol. 37, 1934, 354-386.

[102]The UeR, A. U. - Effect of temperature on the stress-deformation of concrete, Journal of Research (U.S. Nat. Bureau of Standards), RP 970, Vol. 18, Feb. 1937, no 2, 195-204.

[103]HANsen, T. C. - Creep and stress relaxation in concrete, Proc. no 31, Swedish Cement and Concrete Research Institute, Royal Institute of Technology, 1960, Stockholm. 